Endogenous calcitonin gene-related peptide suppresses ischemic brain injuries and progression of cognitive decline

Liuyu ZHAI ${ }^{\mathrm{a}}$, Takayuki SAKURAI ${ }^{\mathrm{a}}$, Akiko KAMIYOSHI ${ }^{\mathrm{a}}$, Yuka ICHIKAWA-SHINDO $^{\mathrm{a}}$, Hisaka KAWATE ${ }^{\mathrm{a}}$, Megumu TANAKA ${ }^{\mathrm{a}}$, Xian XIAN ${ }^{\mathrm{a}}$, Kazutaka HIRABAYASHI ${ }^{\mathrm{a}}$, Kun DAI ${ }^{\mathrm{a}}$, Nanqi CUI ${ }^{\mathrm{a}}$, Keiya TANIMURA ${ }^{\mathrm{a}}$, Teng LIU $^{\mathrm{a}}$, Yangxuan WEI $^{\mathrm{a}}$, Masaaki TANAKA ${ }^{\mathrm{a}}$, Haruka TOMIYAMA ${ }^{\mathrm{a}}$, Akihiro YAMAUCHI ${ }^{\mathrm{b}}$, Kyoko IGARASHI $^{\mathrm{b}}$, Takayuki SHINDO ${ }^{\mathrm{a}}$

${ }^{a}$ Department of Cardiovascular Research, Shinshu University Graduate School of Medicine, Nagano, Japan

bJapan Bio Products Co., Ltd., Tokyo, Japan

\title{
Abbreviated title:
}

Suppression of brain ischemia by CGRP

Word count: 5,283

Number of Figures: 12, Table: 2

\section{Address for correspondence}

Takayuki Shindo, MD, PhD

Department of Cardiovascular Research,

Shinshu University Graduate School of Medicine

Asahi 3-1-1, Matsumoto, Nagano, 390-8621, Japan

Tel: $+81-263-37-2578$

Fax: +81-263-37-3437

Email: tshindo@shinshu-u.ac.jp

Conflicts of interest: The authors have no conflicts of interest 


\section{Sources of funding}

This study was supported by Grants-in-Aid for Scientific Research (KAKENHI), Core Research for Evolutionary Science and Technology (CREST) of Japan Science and Technology Agency (JST) and the Japan Agency for Medical Research and Development (AMED), National Cardiovascular Center research grant for cardiovascular diseases, Grants-in aid of The Public Trust Fund For Clinical Cancer Research, Mitsui Life Social Welfare Foundation, Takeda Science Foundation research grant, Takeda Medical Research Foundation grant, SENSHIN Medical Research Foundation and YOKOYAMA Foundation for Clinical Pharmacology. L.Z received a scholarship from Rotary Yoneyama Memorial Foundation and X.X received a scholarship from Japanese government. 


\begin{abstract}
Calcitonin gene-related peptide (CGRP) is a 37-amino acid peptide and produced by alternative splicing of the transcript of the calcitonin/CGRP gene. Originally identified as a strong vasodilatory and hypotensive peptide, CGRP is now known to be a pleiotropic molecule distributed in various organs, including the brain. In this study, we used CGRP knockout mice (CGRP-/-) to examine the actions of endogenous CGRP during cerebral ischemia. To induce acute and chronic cerebral ischemia, mice were subjected to middle cerebral artery occlusion (MCAO) and bilateral common carotid artery stenosis (BCAS). In the cerebral cortex of wild-type mice (WT), CGRP expression was upregulated after acute infarction. In CGRP-/- subjected to MCAO or BCAS, recovery of cerebral blood flow was slower and exhibited more extensive neuronal cell death. Expression of the inflammatory cytokines was higher in CGRP-/than WT in the acute phase of ischemia. Pathological analysis during the chronic phase revealed more extensive neuronal cell loss and demyelination and higher levels of oxidative stress in CGRP-/- than WT. CGRP-/- also showed less compensatory capillary growth. In an 8-arm radial maze test, CGRP-/- exhibited poorer reference memory than WT. On the other hand, CGRP administration promoted cerebral blood flow recovery after cerebral ischemia. We also found that CGRP directly inhibited the cell death of primary cortical neurons.
\end{abstract}

These results indicate endogenous CGRP is protective against ischemia-induced neuronal cell injury. CGRP could thus be a novel candidate for use in the treatment of both cerebral ischemia and progression of cognitive decline. 


\section{Condensed Abstract}

We used CGRP knockout mice (CGRP-/-) to examine the actions of endogenous CGRP, a strong vasodilatory and hypotensive peptide, during cerebral ischemia. In CGRP-/-, recovery of cerebral blood flow was slower than in wild-type mice. CGRP-/showed more extensive neuronal cell loss, demyelination and poorer memory. Contrary, CGRP administration promoted cerebral blood flow recovery. We also found that CGRP directly inhibited the cell death of primary cortical neurons. These results indicate endogenous CGRP is protective against ischemia-induced neuronal cell injury. CGRP could thus be a novel candidate for use in the treatment of both cerebral ischemia and progression of cognitive decline.

\section{Key Words}

Calcitonin gene-related peptide (CGRP), Cerebral ischemia, Cerebral blood flow, Cognitive decline

\section{Abbreviations}

CGRP: calcitonin gene-related peptide AM: adrenomedullin RAMP: receptor activity-modifying protein CLR: calcitonin receptor-like receptor WT: wild-type

MCAO: middle cerebral artery occlusion

BCAS: bilateral carotid artery stenosis

CBF: cerebral blood flow

BBB: blood-brain barrier

GFAP: glial fibrillary acidic protein 


\section{Introduction}

Cerebrovascular diseases continue to be a major global health problem, especially in aging societies [1], and novel primary prevention strategies and therapeutic approaches are much needed [2]. There are two major types of cerebrovascular diseases: cerebral ischemia and cerebral hemorrhage. Of those, cerebral ischemia accounts for the majority of cases. It is generally accepted that the main cause of cerebral ischemia is thrombosis in situ or embolic occlusion of a cerebral blood vessel, with the latter usually arising from the heart or atherosclerotic plaques in a carotid artery or aortic arch $[3,4]$. The resultant loss of blood flow in the affected blood vessels leads to motor and sensory dysfunction. The mechanisms underlying hypoxic-ischemic brain damage include death of neuronal and glial cells and loss of integrity of the blood-brain barrier (BBB). Cerebral ischemia also increases the risk of developing dementia $[5,6]$. The vascular dementia that often occurs after a stroke is an important aspect for stroke survivors and even minor strokes affect daily functioning [7,8]. Prevention of vascular dementia can be achieved through the prevention and treatment of stroke. Therefore, the early recovery of cerebral blood flow and/or therapy that includes inhibition of apoptosis and maintenance of the BBB may reverse the damage caused by cerebral ischemia and reduce the occurrence of vascular dementia.

Calcitonin gene-related peptide (CGRP) is a 37-amino acid peptide produced as a consequence of alternative RNA processing of the calcitonin gene. Originally identified as a strong vasodilatory neuropeptide, CGRP is primarily released from sensory nerves and is widely distributed in the central and peripheral nervous systems [9-11]. In addition, CGRP is also expressed in the heart, lungs, digestive system, kidney and blood vessels [12-14]. There are two major forms of CGRP, $\alpha$ and $\beta$. $\alpha$ CGRP is predominantly expressed throughout the central and peripheral nervous systems, while $\beta C$ GRP is found mainly in the enteric nervous system $[15,16]$. CGRP exhibits a wide variety of biological effects in addition to its vasodilatory activity. For example, it now appears CGRP is involved in the pathophysiology of migraine [17]. In the heart, CGRP may play a protective role against myocardial ischemia and heart failure $[18,19]$. CGRP also reportedly facilitates angiogenesis in response to ischemia [20,21], improves blood flow and vascular endothelial function [22,23], and plays an important role in inflammation [24,25]. In addition, there is now clear indication that CGRP exerts 
protective effects against ischemia in the brain [26,27]. However, the precise mechanism of its brain protection remains unclear.

CGRP is an adrenomedullin (AM) family peptide and shares the same receptor, calcitonin receptor-like receptor (CLR), with AM. We previously reported the neuroprotective effect of endogenous AM in acute and chronic cerebral ischemia models [28]. To investigate the pathophysiologic significance of endogenous CGRP in cerebral ischemia, in the present study we generated acute and chronic cerebral ischemia models using CGRP knockout mice (CGRP-/-). With this model, we assessed the effect of CGRP deficiency on neuronal cell injury and cognitive decline after cerebral ischemia. 


\section{Materials and methods}

\section{Experimental animals}

CGRP-/- mice were generated by our group using a targeting DNA construct that replaced exon 5, encoding a CGRP-specific region [29]. Healthy male CGRP-/- and their wild-type (WT) littermates were selected for the acute and chronic cerebral ischemia models (10- to 12-week-old male mice for MCAO, 9-week-old male mice for BCAS). All animal manipulations were approved by the Ethics Committee of Shinshu University (Nagano, Japan).

\section{Establishment of middle cerebral artery occlusion (MCAO) model}

Acute cerebral ischemia/reperfusion injury was induced by unilateral middle cerebral artery occlusion (MCAO), as described previously [30]. Male mice between 9 and 12 weeks of age were used. Before the start of the experiments, $1.5 \mathrm{~cm}$ of $6-0$ nylon surgical suture was coated with silicone to prepare an embolic filament. Mice were anesthetized through continuous inhalation of isoflurane, and the common carotid, internal carotid and external carotid arteries of the right side were separated. Blood flow in the external carotid artery was permanently blocked using an electric knife. The common carotid was clipped, and then the embolic filament was inserted from the external carotid artery into the middle cerebral artery when faint resistance was felt. After $2 \mathrm{~h}$ of cerebral ischemia, the nylon line was removed and the incision was sutured. The vessels were reperfused for $22 \mathrm{~h}$, after which the brain tissues were dissected for pathology and gene expression analysis. Cerebral blood flow (CBF) was recorded using an OMEGAZONEOZ-1 laser Doppler blood flow imaging system (Omegawave Inc., Tokyo, Japan). CBF was recorded just before and $2 \mathrm{~h}, 6 \mathrm{~h}$, and $24 \mathrm{~h}$ after the surgery. The CBF values are expressed as percentages of the baseline value.

\section{Establishment of bilateral carotid artery stenosis (BCAS) model}

Chronic cerebral ischemia was induced by bilateral carotid artery stenosis (BCAS), as described previously [31,32]. Male, 9-week-old mice were anesthetized through continuous inhalation of isoflurane, and the common carotid artery was exposed. Two 4-0 silk sutures were then placed loosely around the distal and proximal parts of the right common carotid artery. Using the sutures, the artery was gently lifted, 
and an external microcoil (internal diameter: $0.18 \mathrm{~mm}$ ) was attached just below the carotid bifurcation. In the same manner, a second microcoil of the same size was attached around the left common carotid artery. Mice in the sham group were subjected to the same surgery, but the microcoils were not attached. CBF was recorded using an OMEGAZONEOZ-1 laser Doppler blood flow imaging system prior to the operation, immediately after the operation, $2 \mathrm{~h}$ after the operation, and 1,2, 7, 14, 21, and 28 days after the operation. The CBF values were expressed as percentages of the baseline value.

\section{Behavioral analysis using an 8-arm radial maze test}

To explore the behavioral changes caused by BCAS, behavioral analysis using an 8-arm radial maze (SMART v3.0.00, Panlab, Spain) was conducted as previously reported [28]. The maze consisted of a central platform $(24 \mathrm{~cm}$ in diameter) with 8 arms extending out radially. Before inducing BCAS, each mouse was trained twice per day for 8 days to memorize the apparatus. Food was then withheld for $15 \mathrm{~h}$ prior the test, and the animals were allowed to visit the arms to eat pellets in food cups located near the end of each arm. The performance of the test animals in each trial was assessed using four parameters: (1) working memory errors (numbers of revisits to the same arm over $5 \mathrm{~min}$ ), (2) reference memory errors (numbers of visits to the arm without a food pellet over $5 \mathrm{~min}$ ), (3) Correct answers (numbers of visits to the arm with a food pellet among the initial 4 choices), and (4) required time (the average time required to take all the food pellets). Beginning 28 days after induction of BCAS, we used the same test for 14 days to evaluate changes in behavior. After the behavioral tests, the brain tissues were dissected for pathology and gene expression analysis.

\section{Histology}

The brain tissue dissected from each mouse was fixed overnight in $4 \%$ paraformaldehyde, embedded in paraffin, and cut into $7-\mu \mathrm{m}$-thick sections for histological analysis. The slices were deparaffinized for hematoxylin and eosin (H\&E) staining, Kluver-Barrera staining, TUNEL and immunohistochemical staining. For evaluation of nerve injury, neurofibrils were identified using Bodian's staining. For the immunohistochemical analysis, the brain slices were incubated with rat anti-glial 
fibrillary acidic protein (GFAP; astrocyte marker, Thermo Fisher Scientific, Waltham, MA), mouse anti-8-hydroxy-2'-deoxyguanosine (8-OHdG; DNA oxidation breakdown marker, Japan Institute for the control of Aging, Shizuoka, Japan), and rat anti-mouse CD31 (capillary marker, BD Biosciences, Franklin Lakes, NJ). DAPI (Thermo Fisher Scientific) was used to stain the nuclei.

\section{RNA extraction and quantitative real-time RT-PCR analysis}

Total RNA was extracted from tissues or cells using TRIzol Reagent (Thermo Fisher Scientific), after which the extracted RNA was treated with DNA-Free (Thermo Fisher Scientific) to remove contaminating DNA, and 2- $\mu \mathrm{g}$ samples were reverse transcribed using a High Capacity cDNA Reverse Transcription Kit (Thermo Fisher Scientific). Quantitative real-time RT-PCR was carried out using an Applied Biosystems 7300 real time PCR System with SYBR green (Toyobo, Osaka, Japan) or Realtime PCR Master Mix (Toyobo) and TaqMan probe (MBL, Nagoya, Japan). The primers and probes used are listed in Table 1. Values were normalized to mouse GAPDH (Pre-Developed TaqMan assay reagents, Thermo Fisher Scientific).

\section{Administration of hydralazine to CGRP-/- and WT mice}

CGRP-/- and WT mice were orally administered hydralazine (Sigma-Aldrich, St. Louis, MO, lot $055 \mathrm{~K} 1703$ ) in their drinking water at a dose of $5 \mathrm{mg} / \mathrm{kg} / \mathrm{day}$. Systolic blood pressure was measured in conscious mice using a computerized tail-cuff method (BP-2000; Visitech Systems, Apex, NC). After 4 weeks of hydralazine administration, mice were subjected to BCAS, and $\mathrm{CBF}$ was monitored. After 42 days of BCAS, brain tissues were dissected for pathological analysis.

\section{Continuous administration of CGRP to WT mice}

Eight-week-old male WT mice were subcutaneously administered human $\alpha$ CGRP (Peptide Institute, Inc., Osaka, Japan) at a concentration of $1 \mu \mathrm{M}$ in sterile normal saline using osmotic pumps (Alzet; DORECT Co, Cupertino, CA). The delivery rate was 0.5 $\mu \mathrm{L} / \mathrm{h}$. Systolic blood pressure was monitored throughout the administration. WT mice administered hydralazine in their drinking water at a dose of $5 \mathrm{mg} / \mathrm{kg} /$ day served as controls. After 2 weeks of CGRP or hydralazine administration, mice were subjected to 
BCAS, and CBF was monitored. After 28 days of BCAS, the brain tissues were dissected for pathological and gene expression analysis.

\section{Primary cortical neuron culture}

Cortical neurons were prepared from newborn $\mathrm{C} 57 \mathrm{BL} / 6$ mice as described previously [33]. Briefly, the cerebral cortex was dissected and placed in ice-cold aseptic dissection solution (PBS(-)). After mincing, the tissue was enzymatically digested with 0.1\% DNase I (Sigma-Aldrich, St. Louis, MO) and 1\% trypsin for 5 min at room temperature and then mechanically dissociated. Neurons were seeded into polyD-lysine coated 24-multiwell plates at a density of $1 \times 10^{5}$ viable cells/well. Cultures were maintained in Neurobasal A medium (Thermo Fisher Scientific) supplemented with 5\% FBS, 2\% B27 nutrient (Thermo Fisher Scientific), $0.5 \mathrm{mM}$ L-glutamine, penicillin $(100 \mathrm{U} / \mathrm{ml})$ at $37^{\circ} \mathrm{C}$ in $5 \% \mathrm{CO}_{2}$. After $12 \mathrm{~h}$ of incubation, the medium was changed to serum-free Neurobasal A. We then used RT-PCR to examine expression of CGRP and AM receptor components in primary cortical neurons. The primers used are listed in Table 2. All experiments were carried out on mature neurons grown for 14 days in vitro.

\section{Cell viability assay}

Primary cortical neurons were stimulated with recombinant human TNF- $\alpha$ (200 $\mathrm{ng} / \mathrm{ml}$ ) (Wako, Osaka, Japan) for $72 \mathrm{~h}$ in the presence or absence of graded doses of CGRP. Human $\alpha$ CGRP was added to a concentration of $10^{-9}-10^{-7} \mathrm{M} 1 \mathrm{~h}$ before addition of TNF- $\alpha$. After stimulation with TNF- $\alpha$ for $72 \mathrm{~h}$, the viability of the cortical neurons was assessed using a WST-8 kit (Dojindo, Kumamoto, Japan) according to the manufacturer's instructions. Cells were incubated for $4 \mathrm{~h}$ in WST-8 assay solution, after which the formazan formed was measured as a function of the $\mathrm{OD}_{450}$ using a spectrophotometer.

\section{Statistical analysis}

Values are expressed as means \pm SE. Student's t test was used to determine significant differences between two groups. One-way ANOVA followed by Fisher's PLSD was used to determine significant differences between three or more groups. All 
analyses were performed using SPSS software (v.19). Values of $p<0.05$ were considered significant. 


\section{Results}

CGRP-/- mice show delayed recovery of CBF and more extensive neuronal cell damage after MCAO

We initially generated acute cerebral ischemia injury through MCAO. Laser Doppler images of $\mathrm{CBF}$ before and after the operation are shown in Fig. 1A. Immediately after the operation, both the infarcted and contralateral sides of the brain showed transiently diminished CBF. In both CGRP-/- and WT mice, CBF on the infarcted and contralateral sides were respectively reduced to about $80 \%$ and $60 \%$ of the pre-operation level (Fig. 1B). Although the CBF reduction on the infarcted side immediately after MCAO was nearly the same in WT and CGRP-/- mice, recovery of $\mathrm{CBF}$ at $6 \mathrm{~h}$ and $24 \mathrm{~h}$ showed tendency of reduction in CGRP-/- (Fig. 1C).

Pathological analysis $24 \mathrm{~h}$ after the final CBF measurements showed that CGRP/- brains had more extensive neuronal cell damage in the cerebral cortex, corpus callosum and corpus striatum boundary region; cell density reduction, cellular vacuolation and nuclear alterations were all more apparent in the CGRP-/- than WT mice after MCAO (Fig. 2A-F). In addition, TUNEL analysis showed a higher incidence of apoptosis on the operated side in CGRP-/- than WT mice (Fig. 2G, H), indicating more extensive irreversible cell damage.

Gene expression analysis of the cerebral cortex from WT mice revealed CGRP expression to be significantly higher in the MCAO group than the sham-operation group. In addition, CGRP's receptor, CLR, also tended to be upregulated in the MCAO groups of WT and CGRP-/- mice (Fig. 3A). This suggests CGRP-CLR signaling is involved in the pathogenesis of acute cerebral infarction induced by MCAO. On the other hand, the CGRP receptor's modifying protein, RAMP1, was unchanged or somewhat decreased in the MCAO group. Interestingly, expression of AM, a related peptide, was elevated by MCAO. Expression of AM receptor's modifying protein, RAMP2 and 3, was also elevated by MCAO, which may reflect compensatory upregulation of AM-signaling in brain ischemia. CGRP-/- brains showed significantly increased expression of AM in the cerebral cortex. As AM is a vasodilating peptide, like CGRP, this may reflect compensation for the CGRP deficiency in CGRP-/- brains. Similarly, in the hippocampus, CGRP expression tended to be higher on the operated side in the MCAO group of WT mice (Fig. 3B). AM expression also tended to be 
upregulated in the MCAO groups and was more apparent in CGRP-/- brains.

In addition, in both the cerebral cortex and hippocampus on the operated side in the MACO group, levels of IL-1 $\beta$, IL-6 and TNF- $\langle$, three inflammatory cytokines, were significantly higher in CGRP-/- than WT brains. Taken together, these results indicate that after MCAO, CGRP-/- mice showed extensive neuronal cell damage with greater upregulation of inflammatory cytokines than was seen in WT mice (Fig. 3A, B).

\section{CGRP-/- mice show delayed recovery of CBF and decreased body weight after BCAS}

We next examined the effects of chronic cerebral ischemia in CGRP-/- and WT mice caused by BCAS produced by attaching a coil to bilateral common carotid arteries. Figure 4A shows the time-course of the BCAS analysis. WT and CGRP-/- mice underwent pre-training in an 8-arm radial maze for 8 days before the BCAS operation was performed. After recording the changes in $\mathrm{CBF}$ and body weight for 28 days after placement of the coils, we examined mice's behavior for 14 days using the 8-arm radial maze.

Laser Doppler images of CBF before and after the operation are shown in Fig. 4B. Immediately after the operation, $\mathrm{CBF}$ in $\mathrm{WT}$ and CGRP-/- mice was respectively reduced to about $70 \%$ and $55 \%$ of the pre-BCAS level (Fig. 4C). Two hours after the operation, CBF had recovered to about $80 \%$ in both WT and CGRP-/- mice. In the chronic phase of BCAS, however, CGRP-/- mice showed slower recovery of CBF. Fourteen and 28 days post-BCAS, CBF was significantly lower in CGRP-/- than WT mice (Fig. 4C).

After the BCAS operation, transient body weight reduction was observed in both WT and CGRP-/- mice; however, the effect was more pronounced in CGRP-/- mice. The body weights 2 and 7 days post-BCAS were significantly lower in CGRP-/- than WT mice (Fig. 4D).

CGRP-/- mice show more extensive neuronal cell loss, glial cell activation and less compensatory capillary growth after BCAS.

To analyze the changes early during BCAS, we performed a pathological analysis after 1 or 3 days of BCAS. On day 1 of BCAS, CGRP-/- mice exhibited a greater 
reduction in neuronal cells in the cerebral cortex (Fig. 5A, B) and corpus callosum (Fig. $5 \mathrm{C}, \mathrm{D})$ than WT mice. In addition, numbers of GFAP-positive astrocytes in the corpus striatum boundary region (Fig. 5E, F), cerebral cortex (Fig. 5G, H), and hippocampus (Fig. 5I, J) were higher on day 1 after BCAS in CGRP-/- mice, indicating there was greater activation of glial cells in CGRP-/- than WT mice. On day 3 of BCAS, CGRP-/mice showed less CD31-positive capillary growth in the cerebral cortex (Fig. 5K, L) and corpus callosum (Fig. 5M, N) than WT mice.

CGRP-/- mice show extensive neuronal cell loss, demyelination, astrocyte activation, oxidative stress and less compensatory capillary formation in the chronic stage of BCAS.

To analyze the changes in the chronic stage of BCAS, we performed a pathological analysis after 42 days of BCAS. H\&E staining showed greater neuronal cell loss in the cerebral cortex of CGRP-/- than WT mice (Fig. 6A, B). Kluver-Barrera staining of brain sections revealed that the number of cresyl violet-stained neurons in the cerebral cortex and the amount of luxol fast blue-stained myelin in the corpus striatum boundary region was lower in CGRP-/- than WT brains (Fig. 6C-H), which indicates more extensive neuronal cell loss and demyelination in CGRP-/- brains. CGRP-/- brains also had greater numbers of GFAP-positive astrocytes (Fig. 6I-N), indicating a higher level of astrocyte activation. The levels of oxidative stress were assessed based on the extent of oxidative DNA damage indicated by 8-OHdG immunostaining. We found that 8-OHdG levels were higher in CGRP-/- than WT brains (Fig. 6O, P). In addition, CGRP-/- brains showed less CD31-positive capillary formation in the cerebral cortex and hippocampus dentate gyrus than WT brains (Fig. 6Q-T).

CGRP-/- mice show upregulation of inflammatory cytokines and downregulation of angiogenic factors after BCAS.

Using real-time RT-PCR, we analyzed gene expression after 1, 3, and 42 days of BCAS. The results showed that on day 3 of BCAS in WT mice, CGRP expression was significantly higher in the BCAS group than the sham-operated group. In addition, CGRP-/- mice showed significantly higher CLR expression than WT mice on day 42 of 
BCAS (Fig. 7A). At day 3 and 42 of BCAS, the expression of AM showed tendency of elevation by BCAS in both wild-type and CGRP-/- mice. At day 3 of BCAS, the expressions of RAMP1, RAMP3, and CLR also showed tendency of elevation in both wild-type and CGRP-/- mice. Elevation of RAMP1 and CLR may indicate that CGRPCLR-RAMP1 signaling is involved in the pathogenesis of chronic cerebral infarction induced by BCAS. Elevation of AM, RAMP3 and CLR may represent the compensatory upregulation of AM-CLR-RAMP3 signaling in the subacute phase of BCAS.

Interestingly, cerebrocortical expression of two inflammatory cytokines, IL-6 and TNF- $\alpha$, was significantly higher in CGRP-/- than WT brains on day 1 of BCAS (Fig. 7B). This result is consistent with the observation after MCAO, and indicates that CGRP-/- mice had greater inflammation during the early stage of ischemia after both MCAO and BCAS. In addition, cerebrocortical expression of the angiogenic factors VEGF-A and IGF-1 was lower in CGRP-/- than WT brains on day 3 of BCAS (Fig. 7C). This result is consistent with the observed decrease in capillary formation seen in CGRP-/- brains in the pathological analysis (Fig. 5C, Fig 6E), and indicates that CGRP deficiency may decrease compensative capillary formation after cerebral ischemia.

\section{CGRP-/- mice exhibit increased BBB damage-related factors after BCAS.}

We also assessed expression of BBB damage-related factors on day 42 of BCAS. The levels of AQP4 and MMP9 in the cerebral cortex (Fig. 8A) and the level of AQP4 in the hippocampus (Fig. 8B) were significantly higher in CGRP-/- than WT brains. This suggests CGRP deficiency leads to greater BBB damage during chronic cerebral ischemia.

\section{CGRP-/- mice exhibit greater cognitive disorder after BCAS.}

We used an 8-arm radial maze for behavioral analysis. During 8 days of training before the BCAS operation (training period), there was no difference in the performances of WT and CGRP-/- mice (Fig. 9A). On day 8 of postoperative behavior analysis (day 36 of BCAS), the reference memory error (numbers of visits to the arm without a food pellet over $5 \mathrm{~min}$ ) was significantly higher in CGRP-/- mice, while the correct answers (numbers of visits to the arm with a food pellet during the initial 4 
choices) were significantly lower in CGRP-/- than WT mice (Fig. 9B). These results indicate that CGRP-/- mice had more severe disturbance of memory after BCAS than WT mice.

The difference in blood pressure in CGRP-/- mice does not affect CBF or brain injury

Because CGRP exerts vasodilatory effects, directly measured mean arterial pressure using a catheter inserted into the femoral arteries of mice was reported to be slightly elevated in CGRP-/- [29]. To rule out an effect of the blood pressure difference between WT and CGRP-/- mice, we administered hydralazine (an antihypertensive drug) to both groups and evaluated the CBF and brain injury induced by BCAS in each mouse. Oral administration of hydralazine for 4 weeks in the drinking water (5 $\mathrm{mg} / \mathrm{kg} /$ day) lowered blood pressures and effectively eliminated the difference in blood pressure between the CGRP-/- and WT mice (Fig. 10A). Nevertheless, CBF recovery tended to be slower in CGRP-/- than WT mice (Fig. 10B), and the brain injury was still more severe with greater neuronal cell loss in CGRP-/- mice (Fig. 10C, D). In addition, when we used Bodian's staining to evaluate injury to neurofibrils, we detected greater reductions of neurofibrils in CGRP-/- than WT mice (Fig. 10E, F). These observations indicate that the difference in baseline blood pressures did not account for the delayed CBF recovery and more severe brain injury in CGRP-/- mice.

\section{CGRP administration promotes CBF recovery, suppresses astrocyte activation and increases angiogenesis after cerebral ischemia.}

To further rule out the effect of blood pressure and to verify the protective effects of endogenous CGRP, we administered CGRP or hydralazine to WT mice for 2 weeks prior to BCAS. During the experiment, we monitored the changes in both blood pressure and CBF. Systolic blood pressure was reduced to about $80 \mathrm{mmHg}$ after administration of either hydralazine or CGRP. Before and after the BCAS operation, blood pressures did not differ between the two groups (Fig. 11A). On the other hand, CBF was significantly higher after 7, 14, and 28 of BCAS in the CGRP group than the hydralazine group (Fig. 11B). These results suggest CGRP administration enhances the recovery of $\mathrm{CBF}$ after cerebral ischemia. 
After monitoring blood pressure and CBF for 28 days, we analyzed the pathological changes and gene expression. The pathological results showed that the neuronal cell loss in the cerebral cortex (Fig. 11C, D) and numbers of GFAP-positive astrocytes in the cerebral cortex and corpus callosum were lower in the CGRP group than the hydralazine group (Fig. 11E-H). Moreover, the CGRP group showed greater CD31-positive capillary formation than the hydralazine group (Fig. 11I-L). Thus CGRP administration appears to promote $\mathrm{CBF}$ recovery and capillary formation while suppressing astrocyte activation and inflammatory cytokine expression to reduce neuronal cell injury after cerebral ischemia. And these effects are independent of any change in blood pressure.

\section{CGRP inhibits TNF- $\alpha$-induced apoptosis in primary cortical neuron culture}

Finally, we directly investigated the protective effect of CGRP against neuron death using cultures of primary cortical neurons stimulated for $72 \mathrm{~h}$ with $200 \mathrm{ng} / \mathrm{ml}$ TNF- $\langle$ in the presence or absence of graded doses of CGRP. CGRP was added $1 \mathrm{~h}$ prior to addition of TNF- $\alpha$. RT-PCR was used to identify CGRP receptor components expressed by the cortical neurons. We found that the neurons expressed mRNAs for CLR and its three accessory protein proteins, RAMP1, RAMP2 and RAMP3 (Fig. 12A). Survival of the primary cortical neurons was reduced to about $70 \%$ by TNF- $\alpha$ stimulation, but CGRP $\left(10^{-7} \mathrm{M}\right)$ treatment suppressed that effect (Fig. 12B). 


\section{Discussion}

Despite recent advances in neuroimaging technologies, which enable us to diagnose stoke quickly, brain injury and cognitive impairment induced by stroke continue to be a leading cause of morbidity and mortality. The most common cause of stroke is cerebral ischemia [34], which is characterized by a reduction in cerebral blood flow (CBF) resulting from thromboembolic occlusion of a major cerebral artery or its branches. The reduction of $\mathrm{CBF}$ leads to loss of oxygen and nutrient supply to nerve cells, causing metabolic and functional deficits. In addition, hypertension is the most common risk factor for cerebral ischemia. Consequently, effective control of blood pressure and timely recovery of $\mathrm{CBF}$ to the ischemic regions are very critical for improving stroke prognosis and promoting functional recovery. For that reason, endogenous vasoactive molecules that improve $\mathrm{CBF}$ have attracted our attention. Initially, $\mathrm{AM}$, a pleiotropic vasoactive molecule, appeared to be a promising research target. In past studies, we showed that homozygous knockout (-/-) of either AM or RAMP2 is embryonically lethal due to abnormal vascular development [35,36]. In addition, we reported the neuroprotective effect of endogenous AM in acute and chronic cerebral ischemia models [28]. However, the significance of CGRP, an AM family peptide, in cerebral ischemia, remains unknown. In the present study, therefore, we investigated the pathophysiological significance of CGRP in cerebral ischemia.

In the heart, CGRP exerts a protective effect against cardiac ischemia/reperfusion injury and pressure overload-induced heart failure [19,37]. CGRP is also protective at the onset of vessel injury and remodeling [38], and it plays a protective role against neointimal hyperplasia following wire-induced vascular injury [39]. Collectively, these results are indicative of the vasoprotective actions for CGRP, which contribute to the maintenance of cardiac and vascular homeostasis.

In the present study, we demonstrated that CGRP-deficiency leads to delayed CBF recovery in acute and chronic cerebral ischemia models, which suggests CGRP plays a key role in maintaining CBF. We also found that CGRP gene expression is upregulated on the infarcted side after MCAO and BCAS in WT mice, which suggests this upregulation is a compensatory response to cerebral ischemia. Interestingly, AM gene expression was also upregulated after cerebral ischemia in both WT and CGRP-/- mice, and the elevation was greater in CGRP-/- than WT mice. This suggests the increase in AM is a compensatory response to the CGRP deficiency in CGRP-/- mice. Endogenous 
CGRP thus appears to exert protective effects in an effort to maintain CBF during cerebral ischemia.

CGRP exerts vasodilatory effects, however, in the present study, we demonstrated that the difference in blood pressure in CGRP-/- mice does not affect CBF or brain injury. In addition, from the results of CGRP or hydralazine administration to WT mice, we found that CGRP administration promoted the recovery of CBF and reduced neuronal cell injury, which is independent of any change in blood pressure. Taken together, endogenous CGRP plays a protective role in maintaining CBF and reducing nerve injury independent of blood pressure.

The inflammatory response and oxidative stress that follow cerebral ischemia exacerbates the brain damage and neurological deficits, and they play a central role in the pathogenesis and progression of stroke. It has been reported that CGRP plays a key role in inflammation $[24,25]$, though whether the actions of CGRP are proinflammatory or anti-inflammatory remain controversial. In the present study, we found that expression of inflammatory cytokines was higher in CGRP-/- than WT mice early after induction of ischemia in both the MCAO and BCAS models. This result is consistent with the increased activation of astrocytes in CGRP-/- seen after MCAO and BCAS and suggests CGRP has an anti-inflammatory function after cerebral ischemia, which may be one aspect of its protective effect during cerebral ischemia. In addition, we found higher levels of oxidative stress in CGRP-/- than WT mice after BCAS, suggesting antioxidative effects of CGRP are also involved in the protection against cerebral ischemia. Thus CGRP may protect against cerebral ischemia by suppressing both inflammation and oxidative stress to some extent.

Preventing the occurrence of neuronal cell loss is the ultimate goal of treatment for cerebral ischemia. CGRP reportedly protects cultured smooth muscle cells from apoptosis induced by oxidative stress via activation of ERK1/2 MAPK [40], and CGRP protects cardiomyocytes from hypoxia-induced inflammation and apoptosis by modulating NO production [41]. In addition, we have reported that CGRP exerts a hepatoprotective effect by modulating cytokine expression and preventing apoptosis [42]. In the present study, the incidence of apoptosis and neuronal cell loss after cerebral ischemia was higher in CGRP-/- than WT mice, suggesting CGRP exerts an antiapoptotic effect. To verify the direct beneficial effects of CGRP on neurons cells, we 
induced apoptosis in primary cortical neurons by exposing them to TNF- $\alpha$ in their culture medium with and without CGRP. The result showed that CGRP inhibits TNF- $\alpha$ induced apoptosis in primary cortical neurons, suggesting CGRP directly protects neurons by preventing apoptosis, and this effect would be independent of its beneficial effect on $\mathrm{CBF}$.

Recent studies have shown that new vessel formation after stroke not only replenishes the blood flow to ischemic regions of the brain, but also promotes neurogenesis and improves neurological function. Therefore, drugs that can promote angiogenesis after cerebral ischemia can provide therapeutic benefits during stroke treatment. CGRP reportedly exhibits angiogenic activity in various ischemic disease models [20,21]. In the present study, we found that CGRP-/- mice showed less compensatory capillary growth during chronic cerebral ischemia, suggesting CGRP promotes angiogenesis after cerebral ischemia. However, the specific mechanism remains to be determined.

Vascular dementia is the second most common type of dementia after Alzheimer disease, and stroke increases the risk of this type of dementia. In stroke survivors, the prevalence of vascular dementia is about $30 \%$, and dementia is one of the major cause of dependency after stroke [5]. In contrast to Alzheimer disease, there is great hope that vascular contributions to cognitive impairment can be prevented and treated; that is, effective prevention and treatment of cerebral ischemia is expected to reduce the incidence of dementia. Singh et al. recently reported that CGRP is protective against Alzheimer disease [43]. However, the role of CGRP in vascular dementia induced by cerebral ischemia remains unknown. In the present study, we used an 8-arm radial maze to evaluate the behavioral changes during BCAS in WT and CGRP-/- mice. The result showed that CGRP-/- mice experienced more severe cognitive disorder during BCAS, which suggests CGRP may improve cognitive ability after cerebral ischemia. In addition, we found that factors related to BBB damage were upregulated during BCAS in CGRP-/- mice, indicating disruption of the BBB. Studies have shown there is a significant association between BBB dysfunction and vascular cognitive impairment [44]. Thus, cognitive disorder caused by BCAS in CGRP-/- mice may be in part attributable to damage to the BBB.

Directly measured mean arterial pressure using a catheter inserted into the mice 
femoral arteries was found to be slightly higher in CGRP-/- than WT mice at baseline[29]. To rule out an effect of the blood pressure difference between WT and CGRP-/- mice, in this study, we administered hydralazine to both groups and evaluated the $\mathrm{CBF}$ and brain injury induced by BCAS in each mouse. We observed that $\mathrm{CBF}$ recovery was slower in CGRP-/- than WT mice, and the brain injury was still more severe with greater neuronal cell loss in CGRP-/- mice. These observations indicate that the difference in baseline blood pressures did not contribute much to the poorer cerebral outcomes in CGRP-/- mice.

In summary, we have demonstrated that CGRP contributes to improving CBF recovery after cerebral ischemia. CGRP may also suppress inflammation and oxidative stress, promote angiogenesis and exert a direct anti-apoptotic effect. In addition, CGRP can mitigate the cognitive disorder induced by cerebral ischemia, which is partly attributed to an effect that decreases $\mathrm{BBB}$ damage. These results indicate that endogenous CGRP exerts significant neuroprotective effects during cerebral ischemia, and targeting CGRP may be a promising approach to treating cerebral ischemia and cognitive decline. 


\section{Acknowledgement}

We thank Drs. T. Uemura and K. Tabuchi for explaining the procedure for primary culture of neurons from neonatal mice. 


\section{Figure legends}

\section{Figure 1}

\section{Cerebral blood flow recovery after MCAO}

(A) Representative laser Doppler images of cerebral blood flow (CBF) in WT and CGRP-/- mice before and after middle cerebral artery occlusion (MCAO). $\mathrm{R}$ and $\mathrm{C}$ indicate the rostral and caudal sides. (B) Temporal profile of CBF after MCAO in WT and CGRP-/- mice. CBF in each hemisphere prior to the operation was assigned a value of $100 \%$. Values are expressed as the mean \pm SEM. (C) Comparison of CBF in the ischemic hemisphere (right brain) after MCAO between WT and CGRP-/- mice. $\mathrm{n}=7$ in the WT group and $\mathrm{n}=9$ in the CGRP-/- group.

\section{Figure 2}

\section{Histological changes after MCAO}

(A-F) H\&E staining of the cerebral cortex (A, B), corpus callosum $(\mathrm{C}, \mathrm{D})$ and corpus striatum boundary region (E, F) in WT and CGRP-/- mice $24 \mathrm{~h}$ after MCAO. CGRP-/brains exhibited more extensive neuronal cell damage after MCAO. Scale bar $=100$ $\mu \mathrm{m}$. $(\mathrm{G}, \mathrm{H})$ TUNEL of the cerebral cortex in WT and CGRP-/- mice $24 \mathrm{~h}$ after MCAO. Cells were counter-stained with methyl green; brown staining indicates positive immunoreactivity. CGRP-/- showed greater apoptosis than WT brains. Scale bar $=50$ $\mu \mathrm{m}$. (I) Anatomical schema showing the locations of Figure $2 \mathrm{~A}-\mathrm{H}$.

\section{Figure 3}

\section{Gene expression after MCAO}

Quantitative real-time PCR analysis of the cerebral cortex (A) and hippocampus (B) in WT or CGRP-/- mice after MCAO or sham operation. L and R indicate the left and right cerebral hemispheres, respectively. Data from the uninfarcted hemisphere of shamoperated WT mice was assigned a value of 1 , and values were expressed as the mean \pm SEM. CGRP-/- brains showed significantly increased expression of AM in the cerebral cortex, and IL-1 $\beta$, IL- 6 and TNF- $\alpha$ in the cerebral cortex and hippocampus after MCAO. ${ }^{*} \mathrm{p}<0.05, \mathrm{n}=3-4$ in each group. 


\section{Figure 4}

\section{Cerebral blood flow recovery and body weights after BCAS}

(A) Time-course of the BCAS experiment. (B) Representative laser Doppler images of CBF in WT and CGRP-/- mice before and after BCAS. R and C indicate the rostral and caudal sides. (C) Temporal profile of CBF after BCAS in WT and CGRP-/- mice. CBF in each hemisphere prior to the operation was assigned a value of $100 \%$. Values are expressed as the mean \pm SEM. In WT mice subjected to BCAS, about $80 \%$ recovery of CBF was maintained on day 28 after BCAS. CGRP-/- showed a slower recovery, and CBF on days 14 and 28 after BCAS was significantly lower than in WT mice. ${ }^{*} p<0.05$, $\mathrm{n}=7$ in each group. (D) Temporal profile of body weight after BCAS. In CGRP-/- mice, weight loss after BCAS was observed compared with WT mice. ${ }^{*} p<0.05$, WT BCAS vs CGRP-/- BCAS, $\mathrm{n}=11$ in each group.

\section{Figure 5}

\section{Histological changes early after BCAS}

H\&E staining (A-D) and immunostaining of GFAP-positive astrocytes (E-J) after 1 day of BCAS. CGRP-/- showed a decrease in neuronal cells in cerebral cortex (A, B) and callous corpus (C, D), and an increased in GFAP-positive astrocytes in the corpus striatum boundary region (E, F), cerebral cortex $(\mathrm{G}, \mathrm{H})$, and hippocampus (I, J) after 1 day of BCAS. (K-N) Immunostaining of CD31-positive capillaries after 3 days of BCAS. CGRP-/- showed less CD31-positive capillary formation than WT mice in callous corpus $(\mathrm{K}, \mathrm{L})$ and cerebral cortex $(\mathrm{M}, \mathrm{N})$. Scale bar $=100 \mu \mathrm{m}$. (O) Anatomical schema showing the locations of Figure 5 A-N.

\section{Figure 6}

\section{Histological changes after 42 days of BCAS}

(A, B) H\&E staining of the cerebral cortex. CGRP-/- showed greater neuronal cell loss than WT mice. (C-H) Kluver-Barrera staining of coronal plane sections of whole brain (C, D), cerebral cortex (E, F) and the corpus striatum boundary region (G, H). CGRP-/brains showed extensive neuronal cell loss and demyelination. (I-T) Immunostaining of GFAP-positive astrocytes (I-N); 8OH-dG, a marker of oxidative DNA damage (O, P); and CD31-positive capillaries (Q-T). CGRP-/- brains showed increased astrocyte 
activation, decreased angiogenesis and increased oxidative stress. Scale bar $=100 \mu \mathrm{m}$. (U) Anatomical schema showing the locations of Figure 6 A, B, E-T.

\section{Figure 7}

\section{Cerebrocortical gene expression after BCAS}

Quantitative real-time PCR analysis of the cerebral cortex after 1, 3 and 42 days of BCAS. Gene expression analysis of AM, CGRP and their receptor components (A), inflammatory cytokines (B) and angiogenic makers (C) is shown. Data from the shamoperated WT mice on each day was assigned a value of 1 , and values were expressed as the mean \pm SEM. CGRP-/- mice showed increased expression of IL- 6 and TNF- $\alpha$ and decreased expression of VEGF-A and IGF-1 early after BCAS. ${ }^{*} \mathrm{p}<0.05, \mathrm{n}=3-4$ in each group.

\section{Figure 8}

\section{Expression of blood-brain barrier-related genes after BCAS}

Quantitative real-time PCR analysis of the cerebral cortex (A) and hippocampus (B) after 42 days of BCAS. Data from the sham-operated WT mice were assigned a value of 1 , and values were expressed as the mean \pm SEM. Levels of AQP4 and MMP9 in the cerebral cortex and level of AQP4 in the hippocampus were significantly higher in CGRP-/- than WT brains. ${ }^{*} \mathrm{p}<0.05$ vs WT-BCAS, $\mathrm{n}=4$ in each group.

\section{Figure 9}

\section{Analysis of behavior after BCAS}

(A) Results of 8-arm radial maze test during the training period. The test was performed twice a day for 8 days before BCAS. Values were expressed as the mean \pm SEM. There was no difference between WT and CGRP-/- mice. $n=16$ in each group. (B) Results of 8 -arm radial maze test performed after 28 days of BCAS. Values were expressed as the mean \pm SEM. In the postoperative 8-arm radial maze test, CGRP-/- mice showed more severe memory deficits after BCAS. ${ }^{*} \mathrm{p}<0.05$ vs WT, $\mathrm{n}=11$ in each group.

\section{Figure 10}

Effects of hydralazine administration on systolic blood pressure and CBF recovery 


\section{after BCAS}

(A) Evaluation of systolic blood pressure after administration of hydralazine to CGRP-/and WT mice. Hydralazine administration for 4 weeks lowered blood pressure to the same levels in CGRP-/- and WT mice. (B) Evaluation of CBF recovery after BCAS. Although blood pressures were the same, CBF recovery was slower in CGRP-/- than WT mice. Values were expressed as the mean \pm SEM. $n=3$ in each group. (C-F) H\&E staining of the cerebral cortex $(\mathrm{C}, \mathrm{D})$ and Bodian's staining $(\mathrm{E}, \mathrm{F})$. CGRP-/- showed more extensive neuronal cell loss and reduction of neurofibrils than WT mice. Scale bar in $\mathrm{C}=100 \mu \mathrm{m}, \mathrm{D}=50 \mu \mathrm{m}$. (G) Anatomical schema showing the locations of Figure $10 \mathrm{C}$ F.

\section{Figure 11}

Effects of CGRP or hydralazine administration on systolic blood pressure and CBF recovery after BCAS in WT mice

(A) Evaluation of systolic blood pressure after administration of CGRP or hydralazine in WT mice. There was no significant difference between the hydralazine and CGRP groups. (B) Evaluation of blood flow recovery after BCAS. Values were expressed as the mean \pm SEM. CBF was significantly higher in the CGRP group than the hydralazine group. ${ }^{*} \mathrm{p}<0.05, \mathrm{n}=5$ in the hydralazine group and $\mathrm{n}=7$ in the CGRP group. (C, D) H\&E staining of the cerebral cortex. Neuronal cell loss was reduced in the CGRP group. Scale bar $=100 \mu \mathrm{m}$. (E-L) Immunostaining of GFAP-positive astrocytes (E-H) and CD31-positive capillaries (I-L). In the CGRP group, astrocyte activation was suppressed and angiogenesis was enhanced after BCAS. Scale bar $=100 \mu \mathrm{m}$.

\section{Figure 12}

\section{Protective effect of CGRP against TNF- $\alpha$-induced death in primary cortical neurons}

(A) Total RNA was extracted from primary cortical neurons and subjected to RT-PCR with primers specific for CLR, RAMP1, RAMP2, RAMP3 and Hprt. The expected sizes for the amplified fragments were $788 \mathrm{bp}$ for CLR, $704 \mathrm{bp}$ for RAMP1, $313 \mathrm{bp}$ for RAMP2, $100 \mathrm{bp}$ for RAMP3, and $352 \mathrm{bp}$ for Hprt. (B) Protective effect of CGRP against TNF-<-induced death in primary cortical neurons from newborn C57BL/6 mice. 
Cell viability of cortical neurons was assessed using a WST-8 kit. Data from the control group was assigned a value of 100, and values were expressed as the mean $\pm \mathrm{SEM}$. After $72 \mathrm{~h}$ of stimulation in the presence of $200 \mathrm{ng} / \mathrm{ml} \mathrm{TNF}-\langle$, the cell viability of primary cortical neurons reduced to about $70 \%$. CGRP $\left(10^{-7} \mathrm{M}\right)$ significantly reduced the incidence of cell death induced by TNF-〈treatment. $* \mathrm{p}<0.05, \mathrm{n}=8$ in each group. 


\section{References}

1. Feigin VL, Forouzanfar MH, Krishnamurthi R, Mensah GA, Connor M, Bennett DA, et al. Global and regional burden of stroke during 1990-2010: findings from the Global Burden of Disease Study 2010. Lancet 2014; 383 (9913):245-254.

2. Feigin VL, Norrving B, George MG, Foltz JL, Roth GA, Mensah GA. Prevention of stroke: a strategic global imperative. Nature reviews Neurology 2016; 12 (9):501-512.

3. Marder VJ, Chute DJ, Starkman S, Abolian AM, Kidwell C, Liebeskind D, et al. Analysis of thrombi retrieved from cerebral arteries of patients with acute ischemic stroke. Stroke 2006; 37 (8):2086-2093.

4. Sierra C, Coca A, Schiffrin EL. Vascular mechanisms in the pathogenesis of stroke. Current hypertension reports 2011; 13 (3):200-207.

5. Leys D, Henon H, Mackowiak-Cordoliani MA, Pasquier F. Poststroke dementia. The Lancet Neurology 2005; 4 (11):752-759.

6. Moskowitz MA, Lo EH, Iadecola C. The science of stroke: mechanisms in search of treatments. Neuron 2010; 67 (2):181-198.

7. Du SQ, Wang XR, Xiao LY, Tu JF, Zhu W, He T, et al. Molecular Mechanisms of Vascular Dementia: What Can Be Learned from Animal Models of Chronic Cerebral Hypoperfusion? Molecular neurobiology 2016.

8. Fride Y, Adamit T, Maeir A, Ben Assayag E, Bornstein NM, Korczyn AD, et al. What are the correlates of cognition and participation to return to work after first ever mild stroke? Topics in stroke rehabilitation 2015; 22 (5):317-325.

9. Eftekhari S, Salvatore CA, Johansson S, Chen TB, Zeng Z, Edvinsson L. Localization of CGRP, CGRP receptor, PACAP and glutamate in trigeminal ganglion. Relation to the blood-brain barrier. Brain research 2015; 1600:93-109.

10. Kruger L, Mantyh PW, Sternini C, Brecha NC, Mantyh CR. Calcitonin gene-related peptide (CGRP) in the rat central nervous system: patterns of immunoreactivity and receptor binding sites. Brain research 1988; 463 (2):223-244.

11. Rosenfeld MG, Mermod JJ, Amara SG, Swanson LW, Sawchenko PE, Rivier J, et al. Production of a novel neuropeptide encoded by the calcitonin gene via tissue-specific RNA processing. Nature 1983; 304 (5922):129-135.

12. Kawasaki H, Takasaki K, Saito A, Goto K. Calcitonin gene-related peptide acts as a 
novel vasodilator neurotransmitter in mesenteric resistance vessels of the rat. Nature $1988 ; 335$ (6186):164-167.

13. Uddman R, Edvinsson L, Ekblad E, Hakanson R, Sundler F. Calcitonin gene-related peptide (CGRP): perivascular distribution and vasodilatory effects. Regulatory peptides $1986 ; 15(1): 1-23$.

14. Villarreal D, Reams G, Freeman R. Calcitonin gene-related peptide and the kidney. Current opinion in nephrology and hypertension 1994; 3 (4):453-458.

15. Matteoli M, Haimann C, Torri-Tarelli F, Polak JM, Ceccarelli B, De Camilli P. Differential effect of alpha-latrotoxin on exocytosis from small synaptic vesicles and from large dense-core vesicles containing calcitonin gene-related peptide at the frog neuromuscular junction. Proceedings of the National Academy of Sciences of the United States of America 1988; 85 (19):7366-7370.

16. Rezaeian $\mathrm{AH}$, Isokane $\mathrm{T}$, Nishibori $\mathrm{M}$, Chiba $\mathrm{M}$, Hiraiwa $\mathrm{N}$, Yoshizawa $\mathrm{M}$, et al. alphaCGRP and betaCGRP transcript amount in mouse tissues of various developmental stages and their tissue expression sites. Brain \& development 2009; 31 (9):682-693.

17. Ramos-Romero ML, Sobrino-Mejia FE. [Calcitonin gene-related peptide: a key player neuropeptide in migraine]. Revista de neurologia 2016; 63 (10):460-468.

18. Chai W, Mehrotra S, Jan Danser AH, Schoemaker RG. The role of calcitonin generelated peptide (CGRP) in ischemic preconditioning in isolated rat hearts. European journal of pharmacology 2006; 531 (1-3):246-253.

19. Li J, Levick SP, DiPette DJ, Janicki JS, Supowit SC. Alpha-calcitonin gene-related peptide is protective against pressure overload-induced heart failure. Regulatory peptides 2013; 185:20-28.

20. Mishima T, Ito Y, Hosono K, Tamura Y, Uchida Y, Hirata M, et al. Calcitonin generelated peptide facilitates revascularization during hindlimb ischemia in mice. American journal of physiology Heart and circulatory physiology 2011; 300 (2):H431-439.

21. Zheng S, Li W, Xu M, Bai X, Zhou Z, Han J, et al. Calcitonin gene-related peptide promotes angiogenesis via AMP-activated protein kinase. American journal of physiology Cell physiology 2010; 299 (6):C1485-1492.

22. Just S, Arndt K, Doods H. The role of CGRP and nicotinic receptors in centrally evoked facial blood flow changes. Neuroscience letters 2005; 381 (1-2):120-124. 
23. Wang Z, Martorell BC, Walchli T, Vogel O, Fischer J, Born W, et al. Calcitonin gene-related peptide (CGRP) receptors are important to maintain cerebrovascular reactivity in chronic hypertension. PloS one 2015; 10 (4):e0123697.

24. Fernandes ES, Schmidhuber SM, Brain SD. Sensory-nerve-derived neuropeptides: possible therapeutic targets. Handbook of experimental pharmacology 2009; (194):393416.

25. Permpoonputtana K, Porter JE, Govitrapong P. Calcitonin gene-related peptide mediates an inflammatory response in Schwann cells via cAMP-dependent ERK signaling cascade. Life sciences 2016; 144:19-25.

26. Liu Z, Liu Q, Cai H, Xu C, Liu G, Li Z. Calcitonin gene-related peptide prevents blood-brain barrier injury and brain edema induced by focal cerebral ischemia reperfusion. Regulatory peptides 2011; 171 (1-3):19-25.

27. Yang SI, Yuan Y, Jiao S, Luo QI, Yu J. Calcitonin gene-related peptide protects rats from cerebral ischemia/reperfusion injury via a mechanism of action in the MAPK pathway. Biomedical reports 2016; 4 (6):699-703.

28. Igarashi K, Sakurai T, Kamiyoshi A, Ichikawa-Shindo Y, Kawate H, Yamauchi A, et al. Pathophysiological roles of adrenomedullin-RAMP2 system in acute and chronic cerebral ischemia. Peptides 2014; 62:21-31.

29. Oh-hashi Y, Shindo T, Kurihara Y, Imai T, Wang Y, Morita H, et al. Elevated sympathetic nervous activity in mice deficient in alphaCGRP. Circulation research 2001; 89 (11):983-990.

30. Shah ZA, Namiranian K, Klaus J, Kibler K, Dore S. Use of an optimized transient occlusion of the middle cerebral artery protocol for the mouse stroke model. J Stroke Cerebrovasc Dis 2006; 15 (4):133-138.

31. Maki T, Ihara M, Fujita Y, Nambu T, Harada H, Ito H, et al. Angiogenic roles of adrenomedullin through vascular endothelial growth factor induction. Neuroreport 2011; 22 (9):442-447.

32. Shibata M, Ohtani R, Ihara M, Tomimoto H. White matter lesions and glial activation in a novel mouse model of chronic cerebral hypoperfusion. Stroke 2004; 35 (11):2598-2603.

33. Uemura T, Mori H, Mishina M. Direct interaction of GluRdelta2 with Shank scaffold proteins in cerebellar Purkinje cells. Molecular and cellular neurosciences 
2004; 26 (2):330-341.

34. Feigin VL. Stroke epidemiology in the developing world. Lancet 2005; 365 (9478):2160-2161.

35. Shindo T, Kurihara Y, Nishimatsu H, Moriyama N, Kakoki M, Wang Y, et al. Vascular abnormalities and elevated blood pressure in mice lacking adrenomedullin gene. Circulation 2001; 104 (16):1964-1971.

36. Ichikawa-Shindo Y, Sakurai T, Kamiyoshi A, Kawate H, Iinuma N, Yoshizawa T, et al. The GPCR modulator protein RAMP2 is essential for angiogenesis and vascular integrity. J Clin Invest 2008; 118 (1):29-39.

37. Zheng LR, Han J, Yao L, Sun YL, Jiang DM, Hu SJ, et al. Up-regulation of calcitonin gene-related peptide protects streptozotocin-induced diabetic hearts from ischemia/reperfusion injury. International journal of cardiology 2012; 156 (2):192-198.

38. Brain SD, Grant AD. Vascular actions of calcitonin gene-related peptide and adrenomedullin. Physiological reviews 2004; 84 (3):903-934.

39. Yang L, Sakurai T, Kamiyoshi A, Ichikawa-Shindo Y, Kawate H, Yoshizawa T, et al. Endogenous CGRP protects against neointimal hyperplasia following wire-induced vascular injury. Journal of molecular and cellular cardiology 2013; 59:55-66.

40. Schaeffer C, Vandroux D, Thomassin L, Athias P, Rochette L, Connat JL. Calcitonin gene-related peptide partly protects cultured smooth muscle cells from apoptosis induced by an oxidative stress via activation of ERK1/2 MAPK. Biochimica et biophysica acta 2003; 1643 (1-3):65-73.

41. Duan L, Lei H, Zhang Y, Wan B, Chang J, Feng Q, et al. Calcitonin Gene-Related Peptide Improves Hypoxia-Induced Inflammation and Apoptosis via Nitric Oxide in H9c2 Cardiomyoblast Cells. Cardiology 2016; 133 (1):44-53.

42. Kamiyoshi A, Sakurai T, Ichikawa-Shindo Y, Fukuchi J, Kawate H, Muto S, et al. Endogenous alphaCGRP protects against concanavalin A-induced hepatitis in mice. Biochemical and biophysical research communications 2006; 343 (1):152-158.

43. Singh Y, Gupta G, Shrivastava B, Dahiya R, Tiwari J, Ashwathanarayana M, et al. Calcitonin gene-related peptide (CGRP): A novel target for Alzheimer's disease. CNS neuroscience \& therapeutics 2017.

44. Toyama K, Spin JM, Tsao PS. Role of microRNAs on Blood Brain Barrier Dysfunction in Vascular Cognitive Impairment. Current drug delivery 2016. 
A
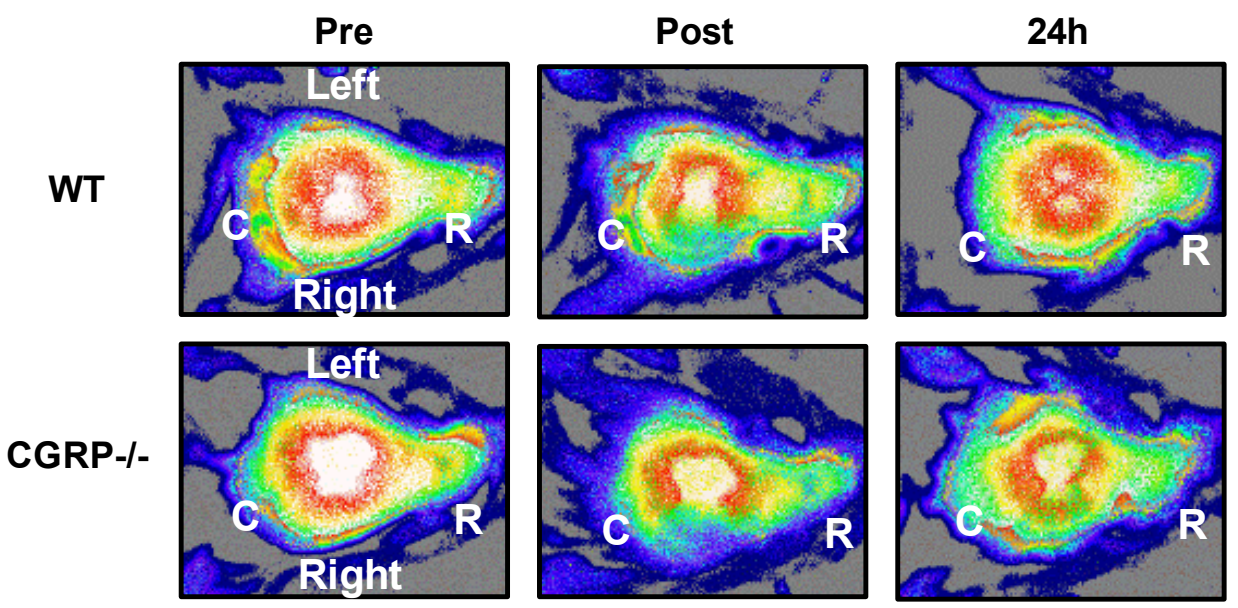

C: Caudal side

R: Rostral side
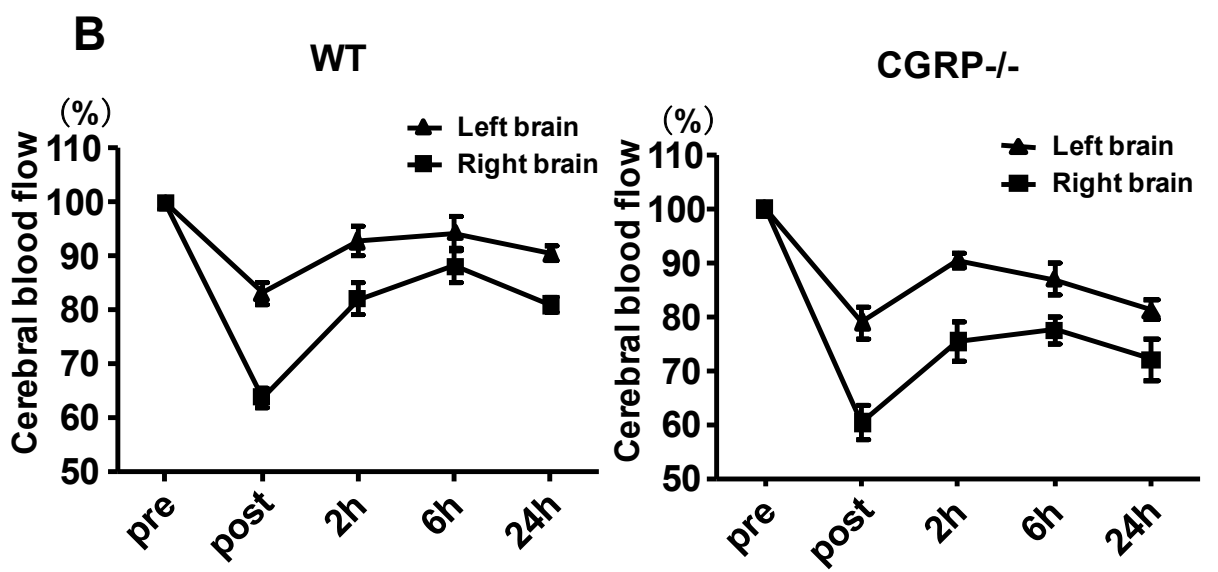

C Wild-type vs. CGRP-/-

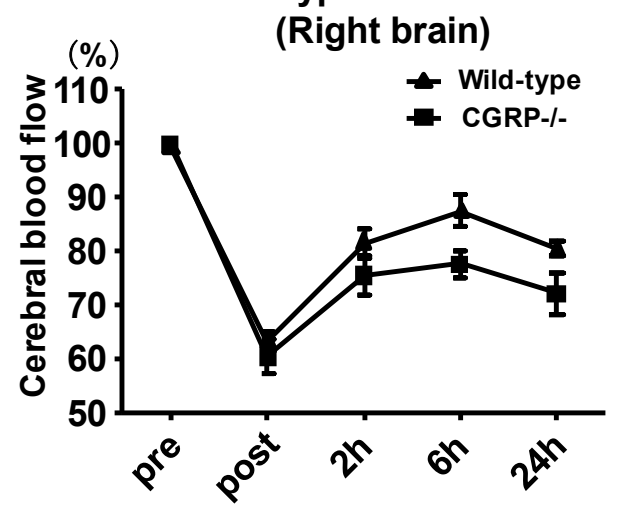

Figure 1 


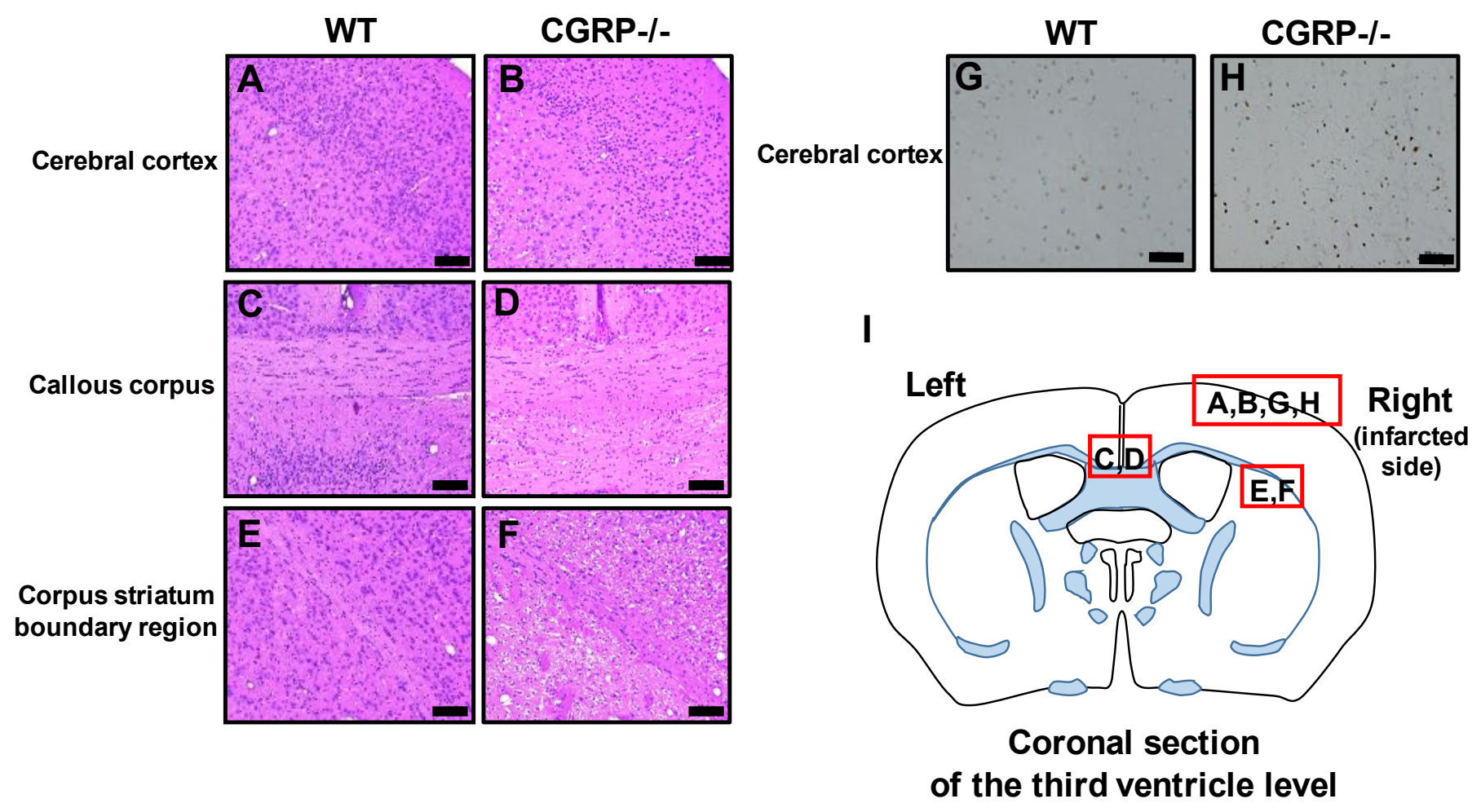

Figure 2 

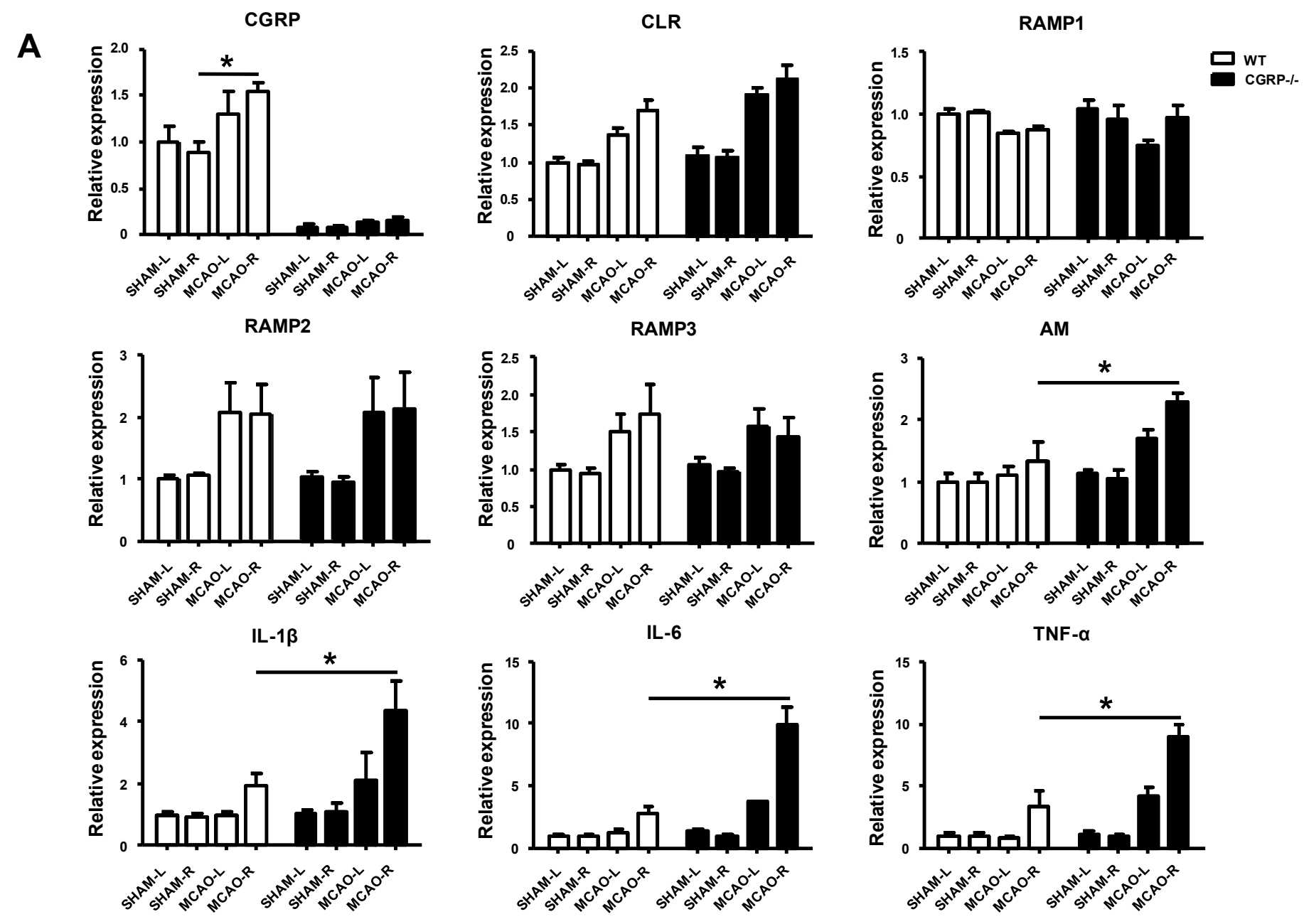

Figure 3 

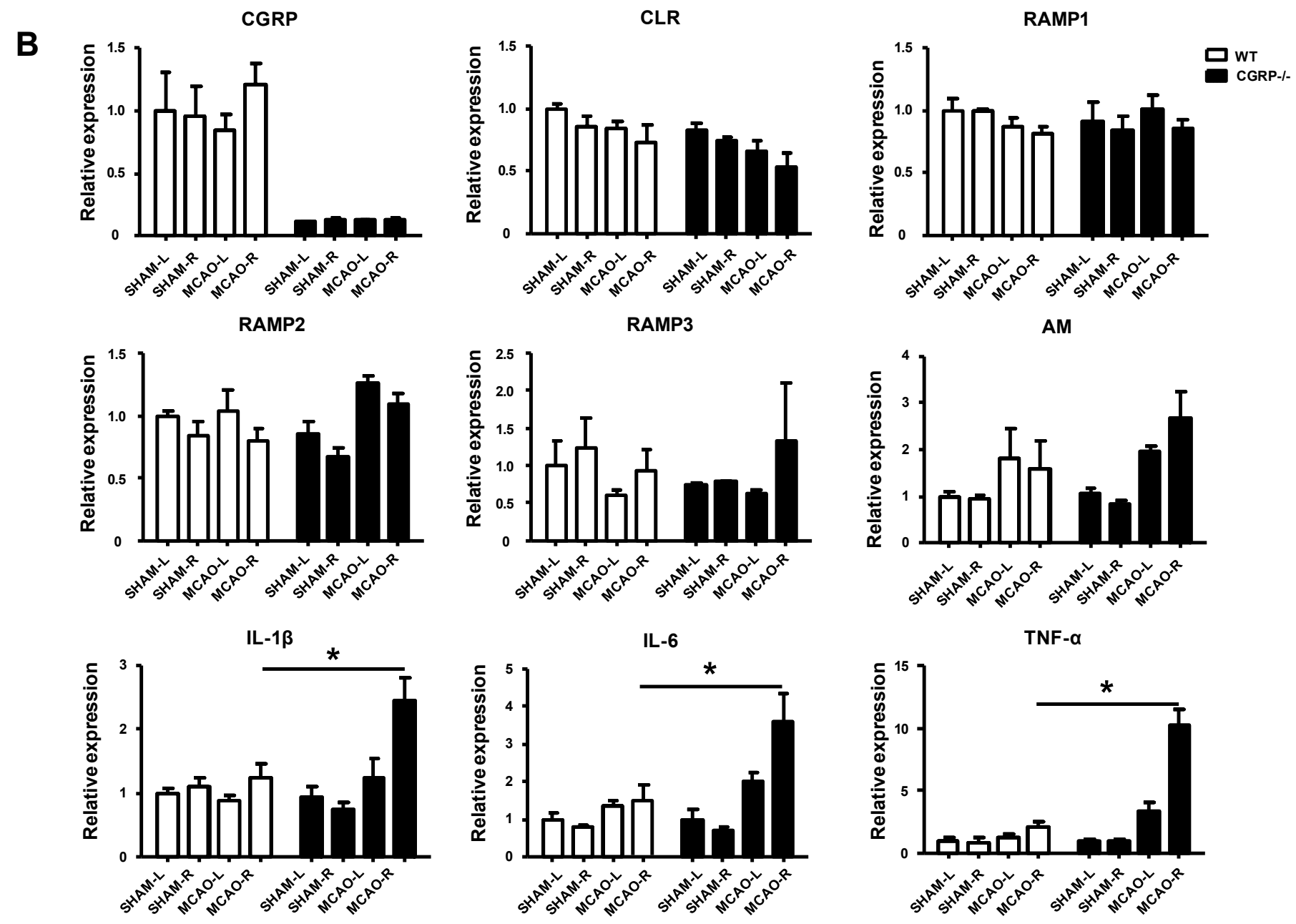

Figure 3 
A

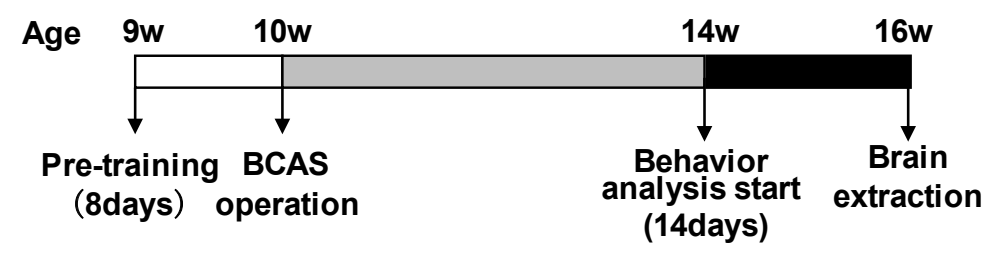

B

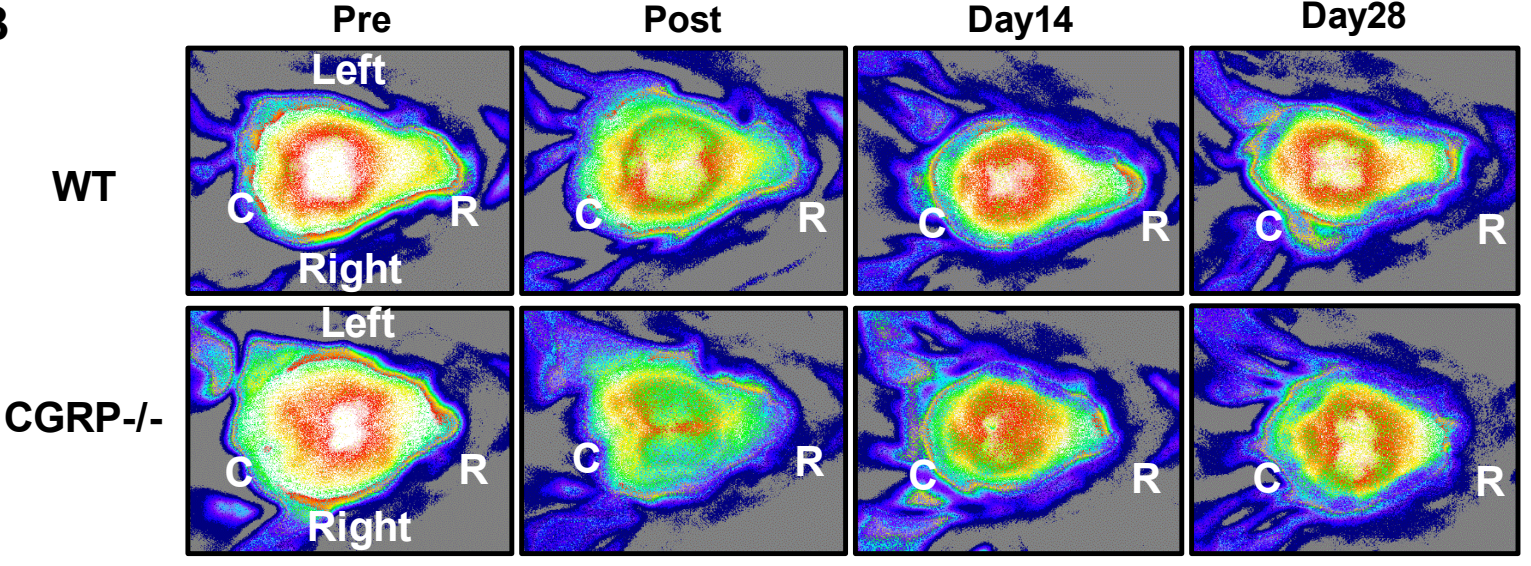

Figure 4 

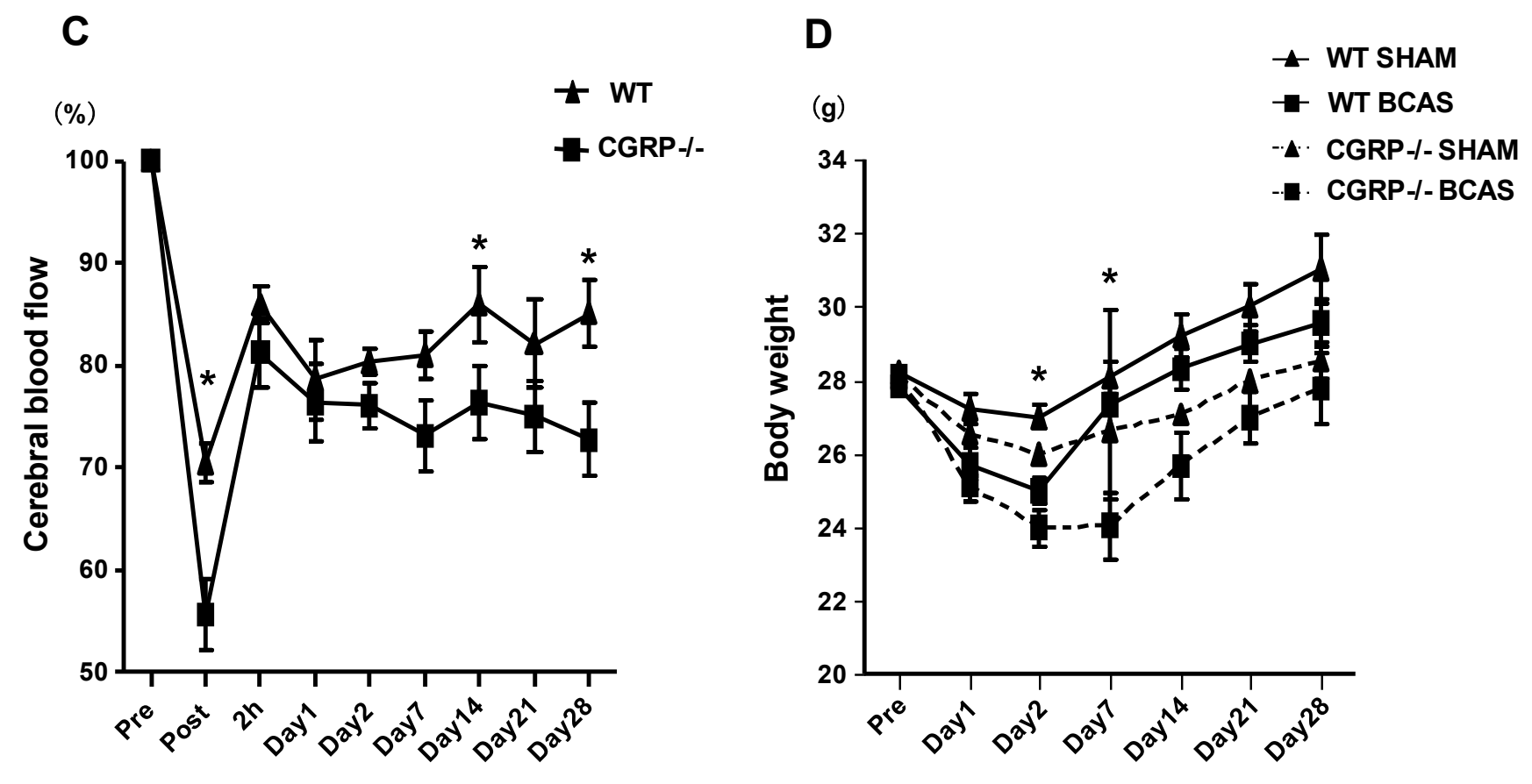

Figure 4 

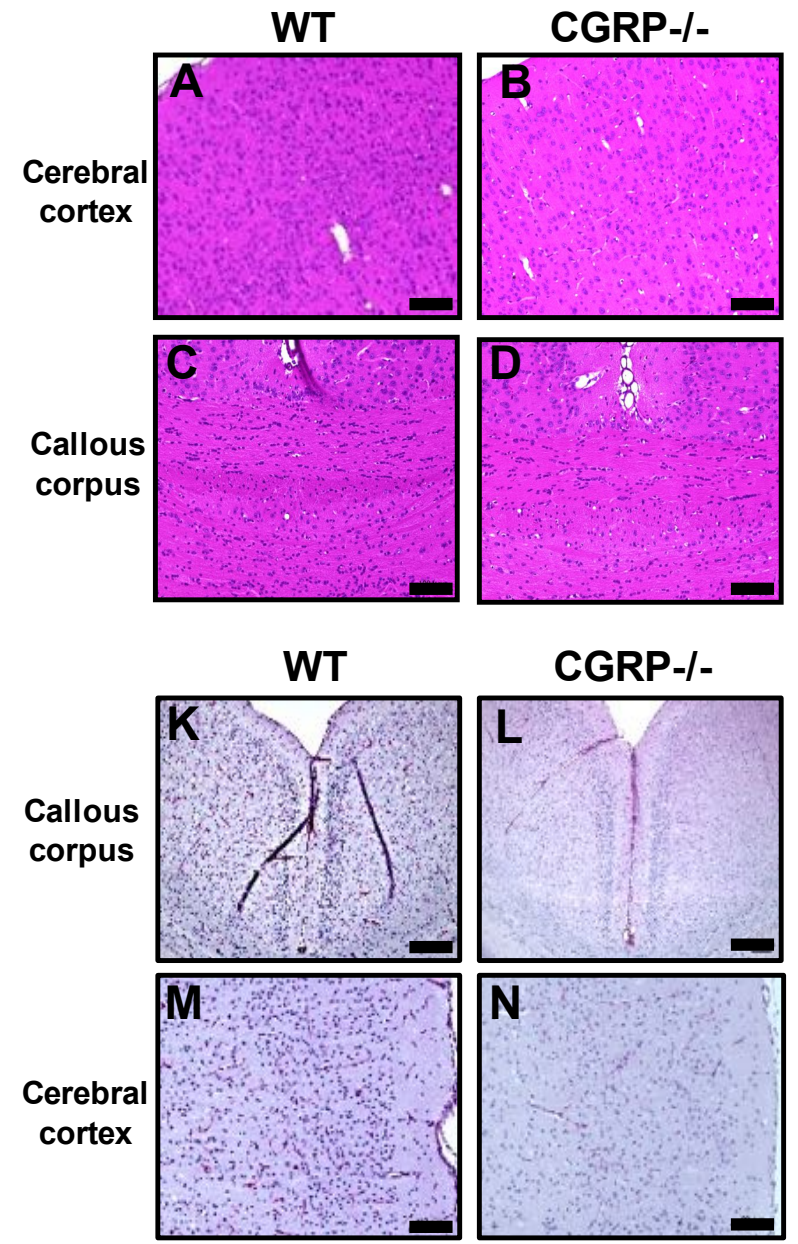

CGRP-/-

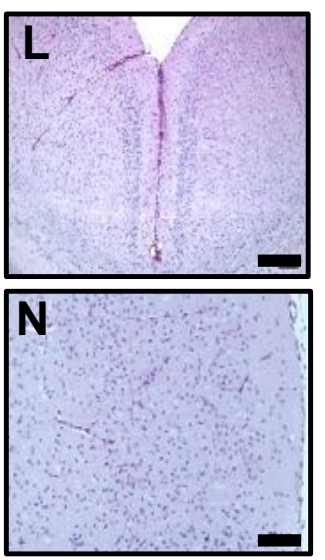

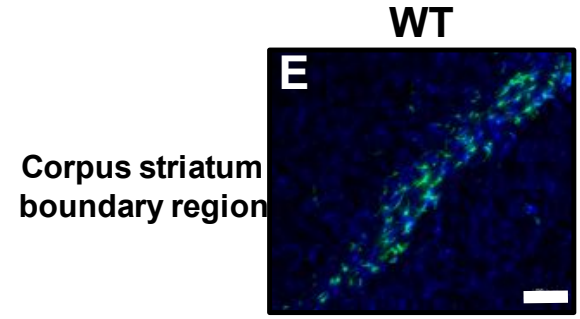

CGRP-/-
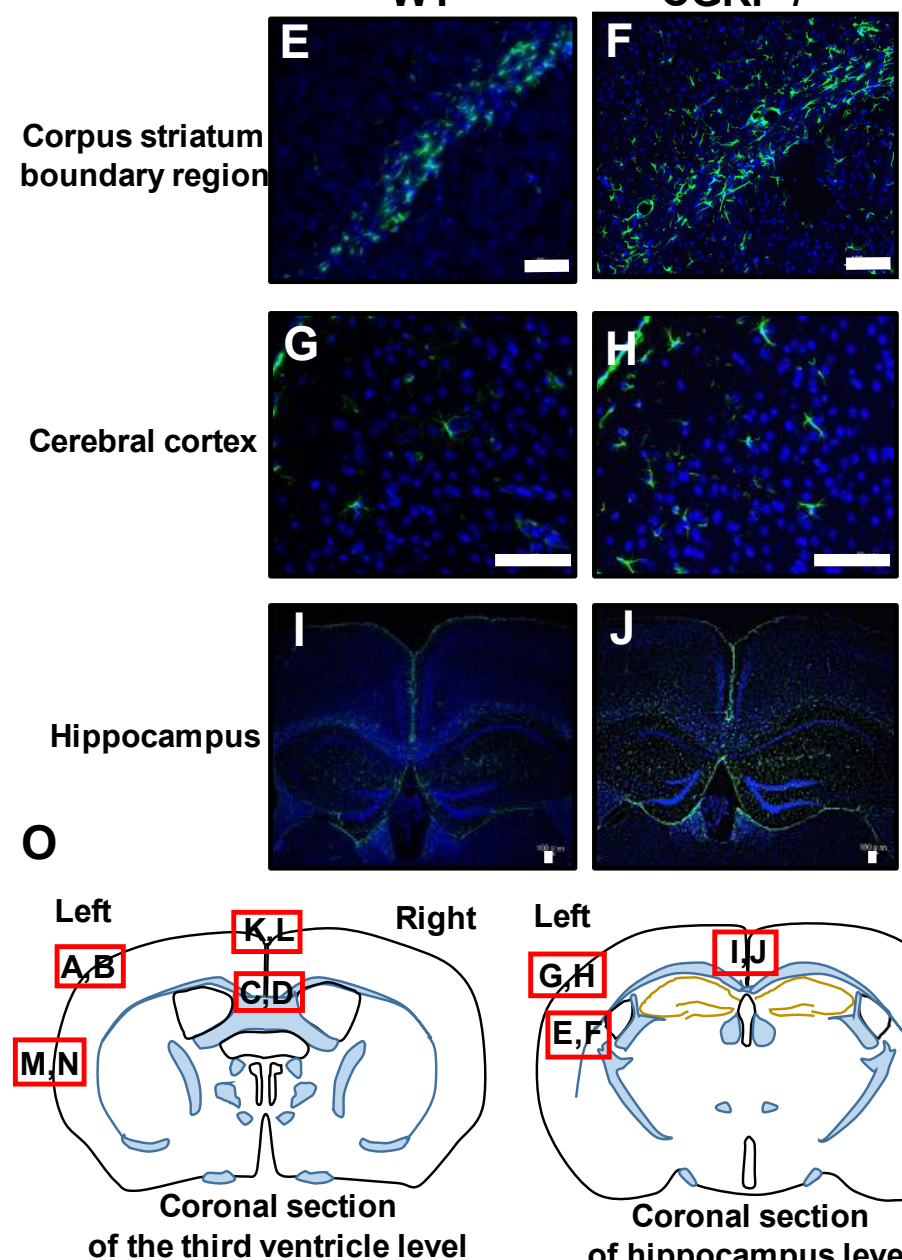

Left

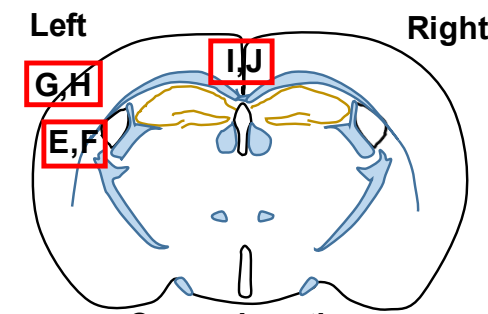

Coronal section of hippocampus level

Figure 5 


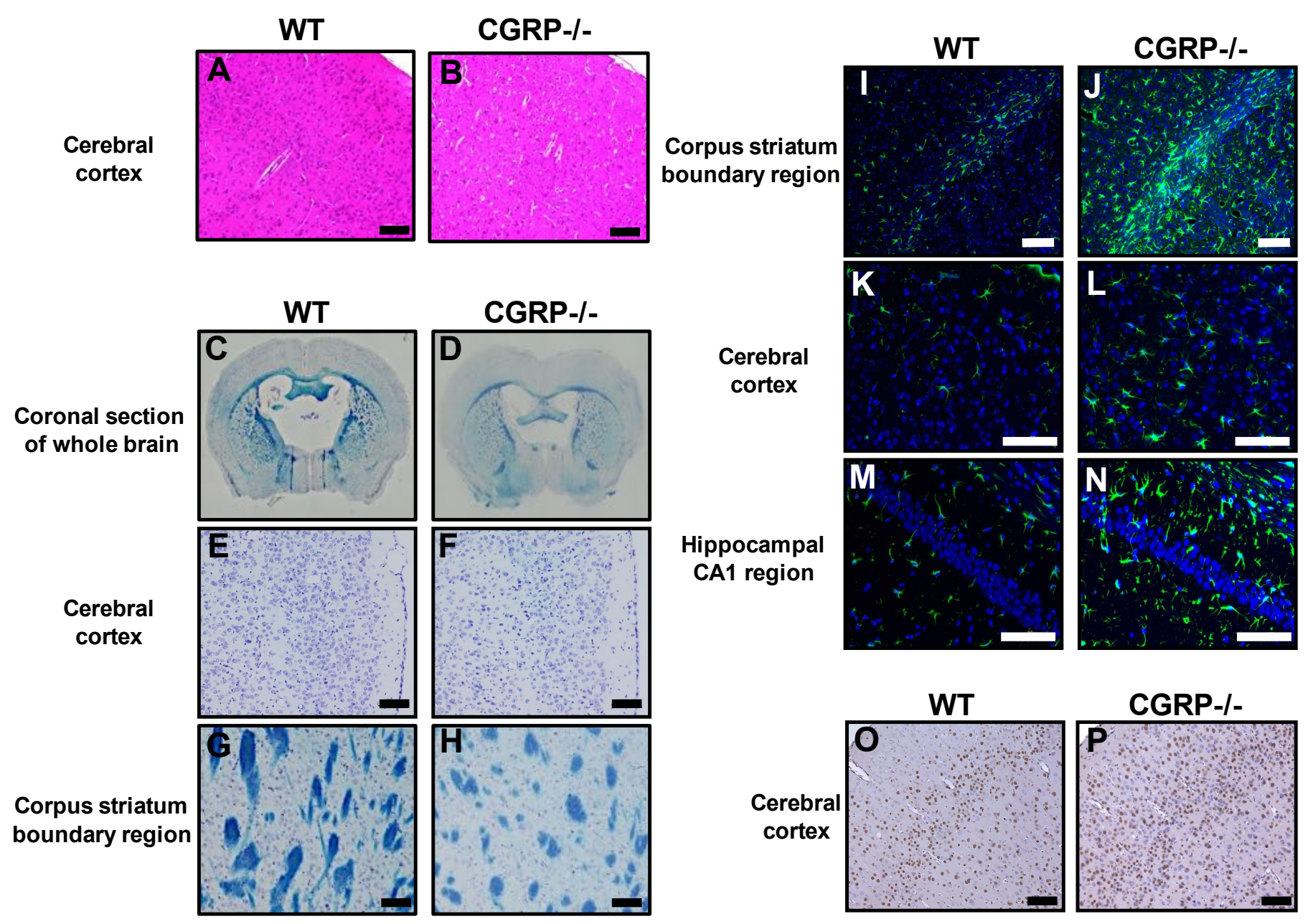

Figure 6 
U

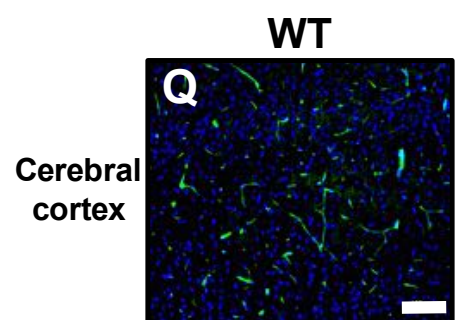

\section{CGRP-/-}
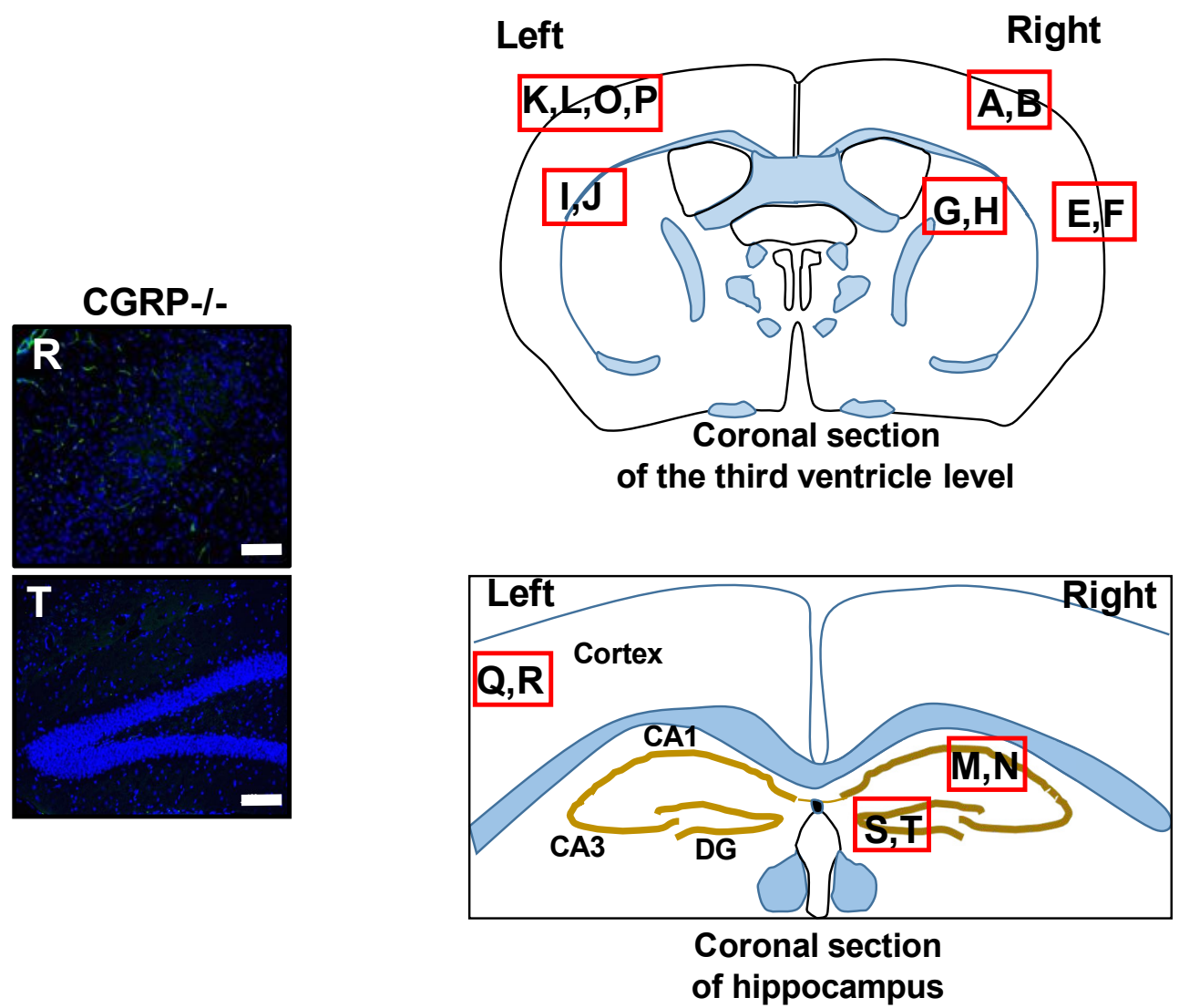

Figure 6 
A
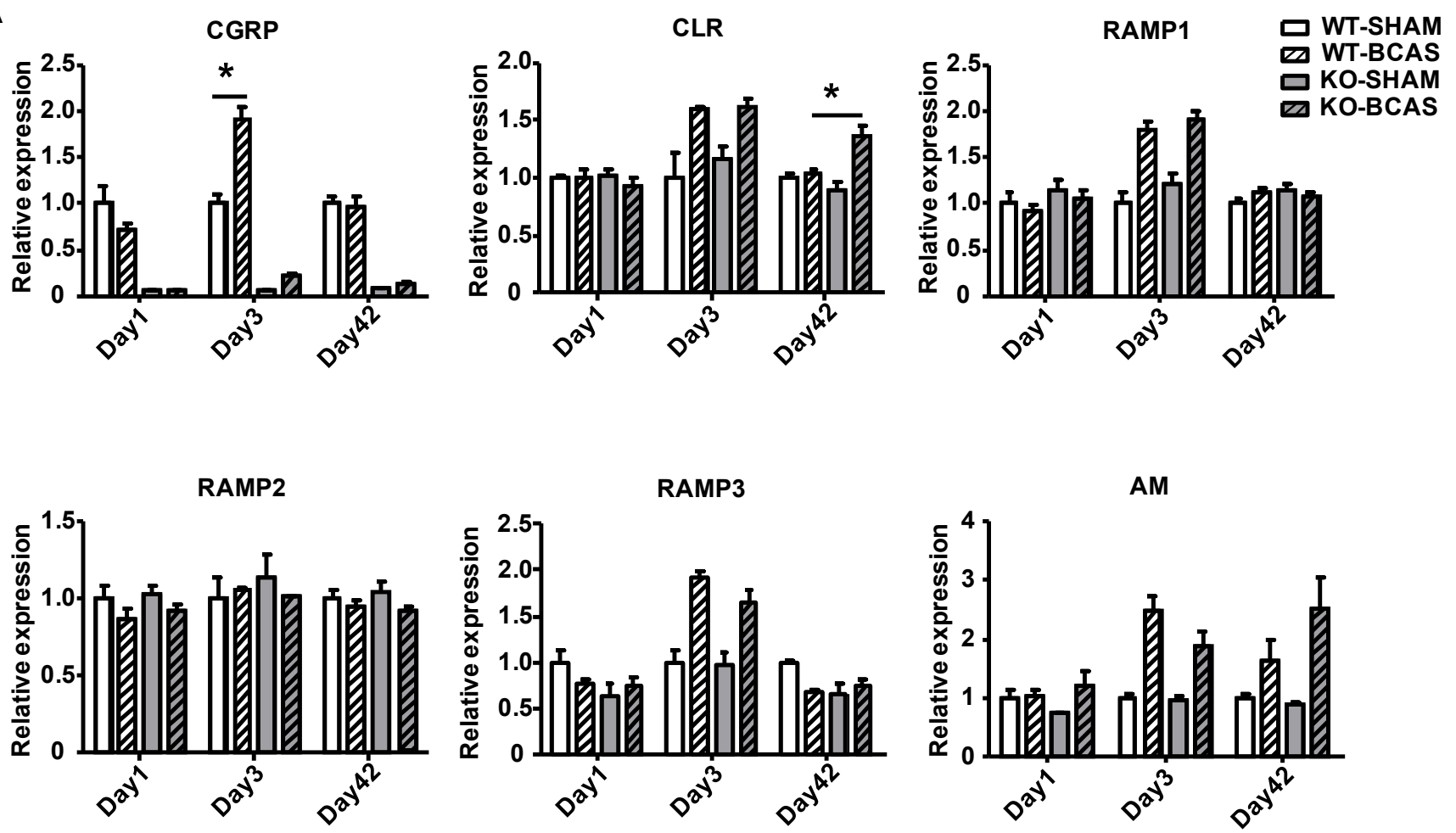

Figure 7 
B
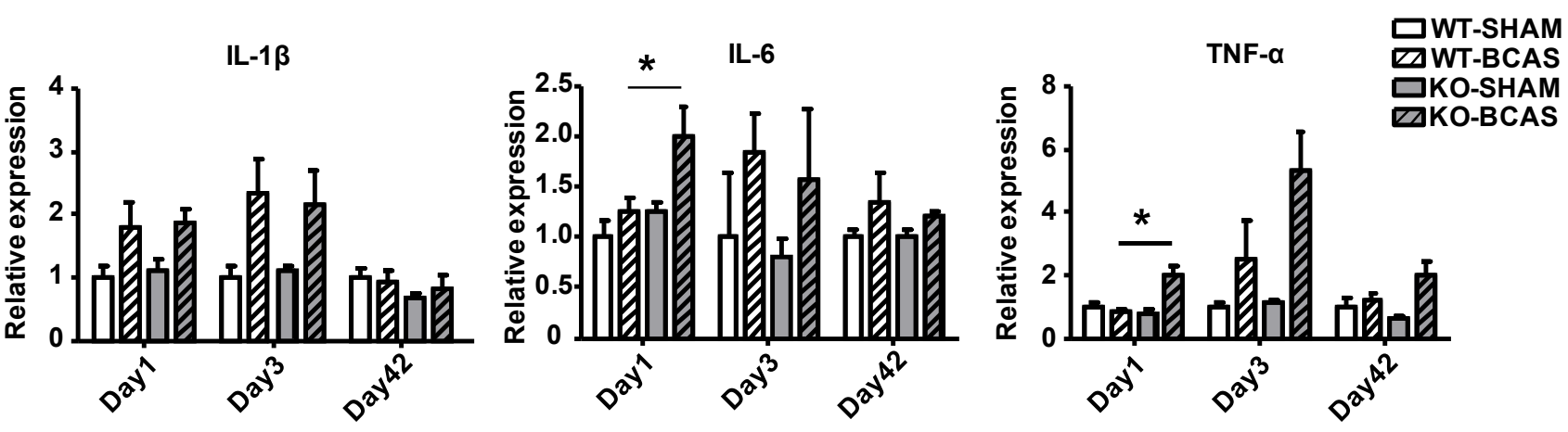

C
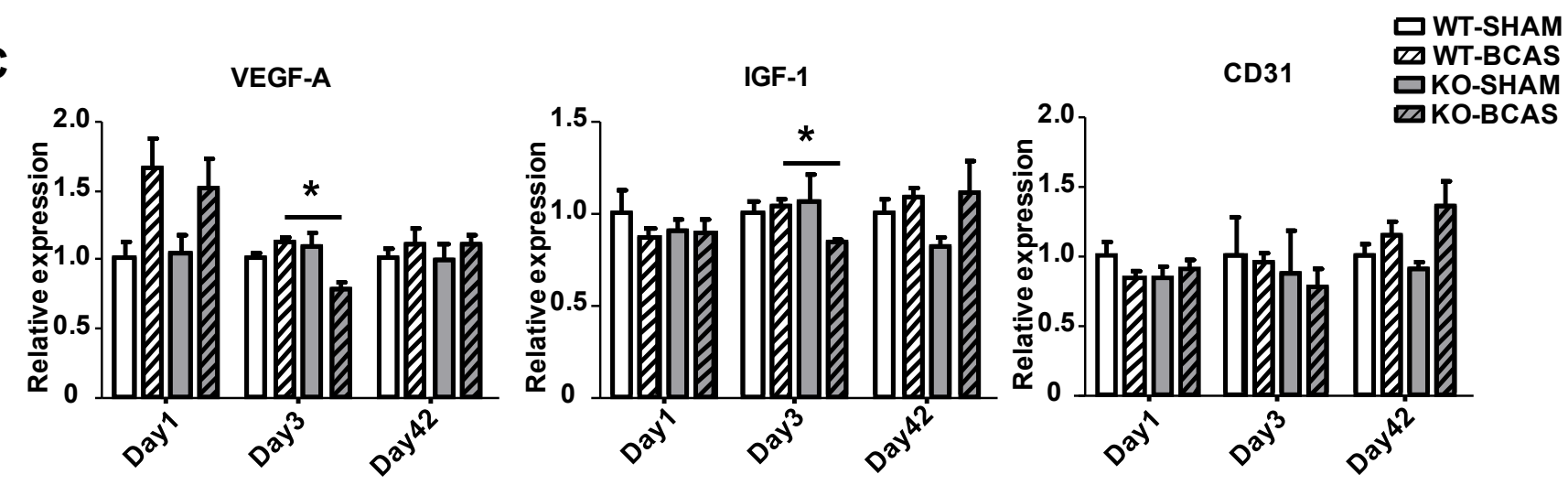

Figure 7 

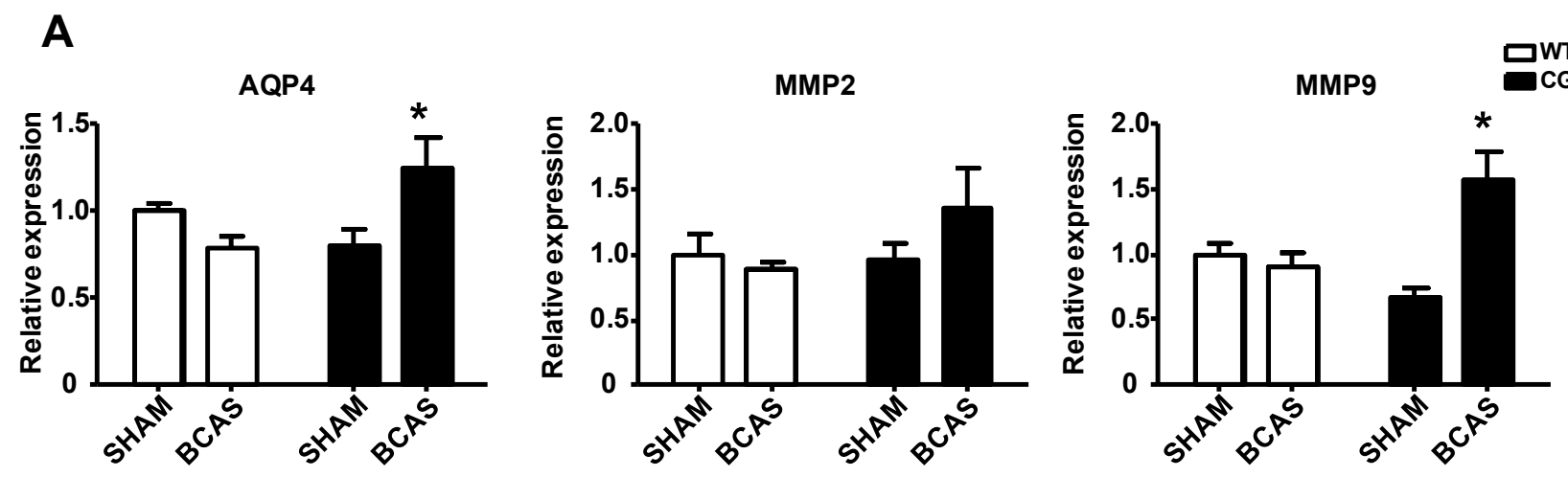

B
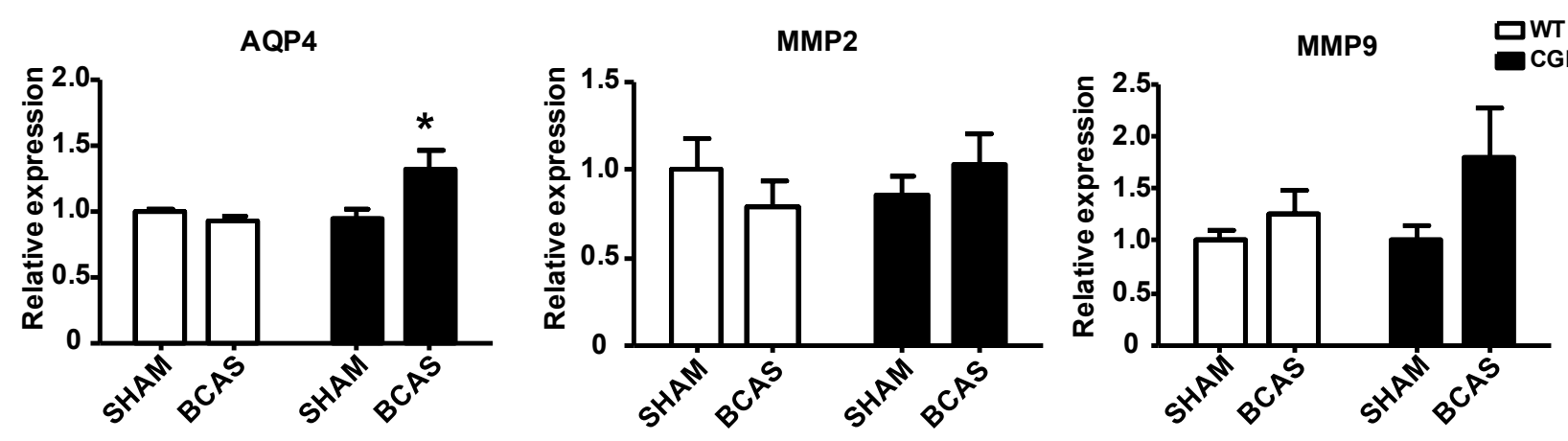

Figure 8 
A
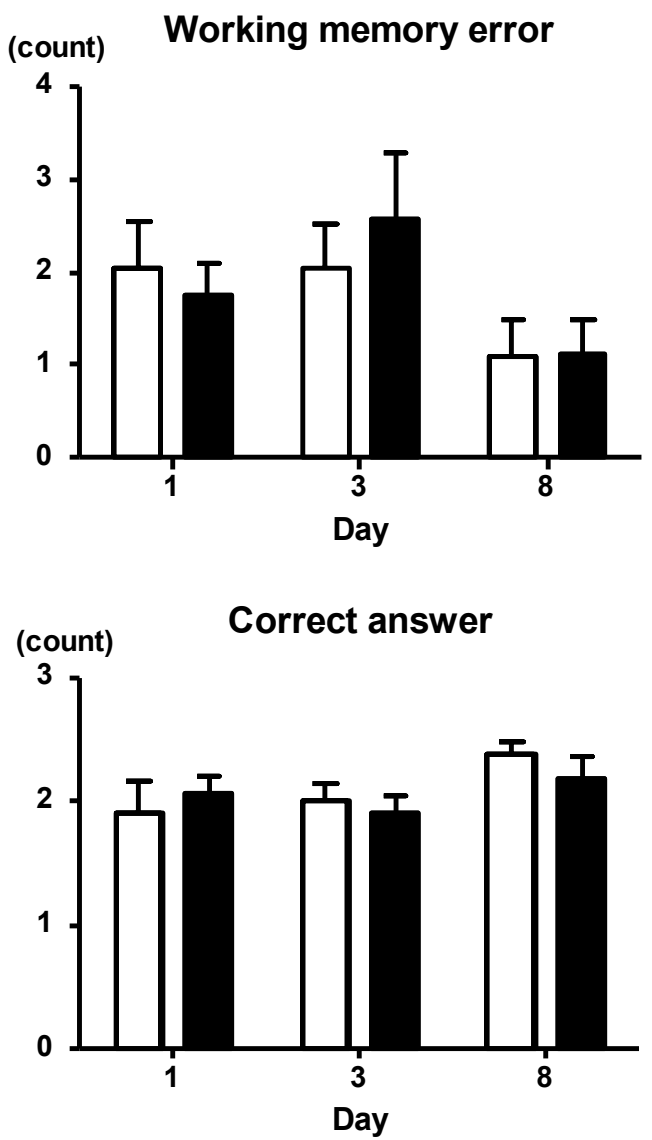
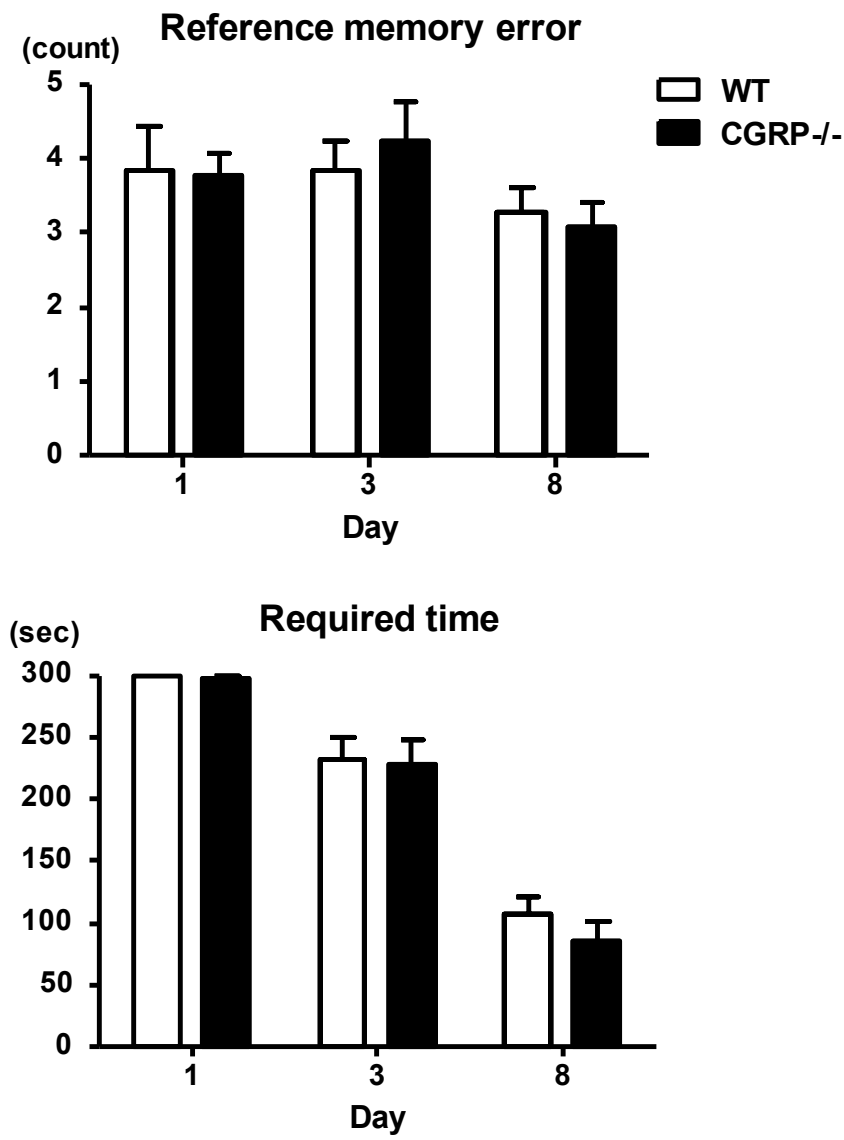

Figure 9 
B
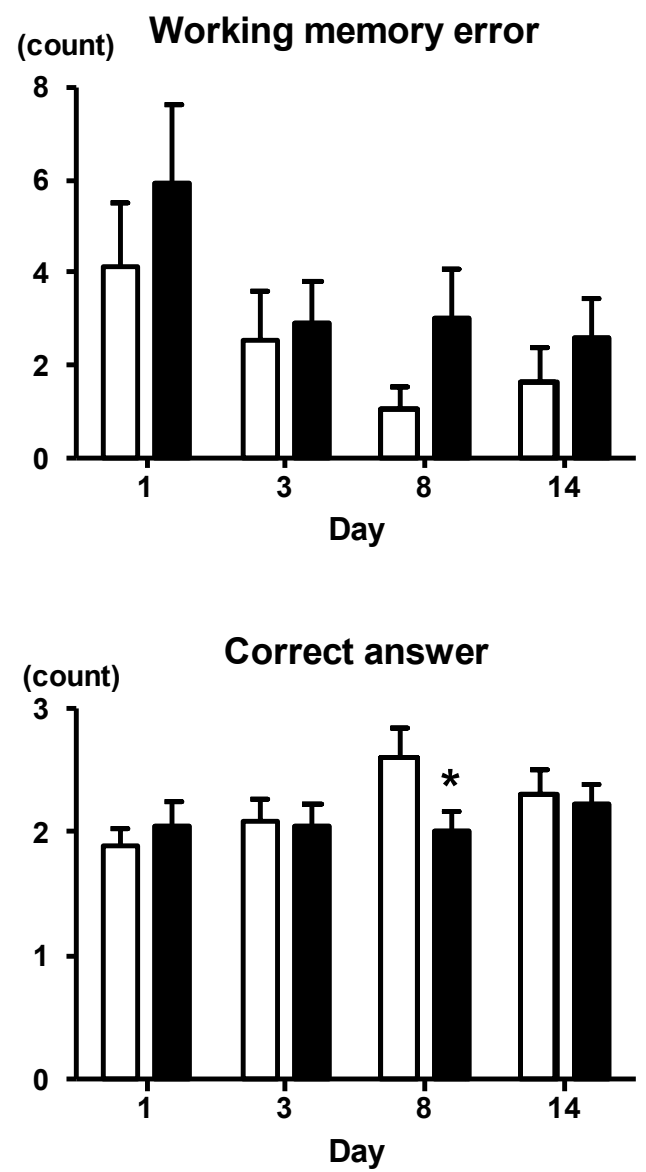
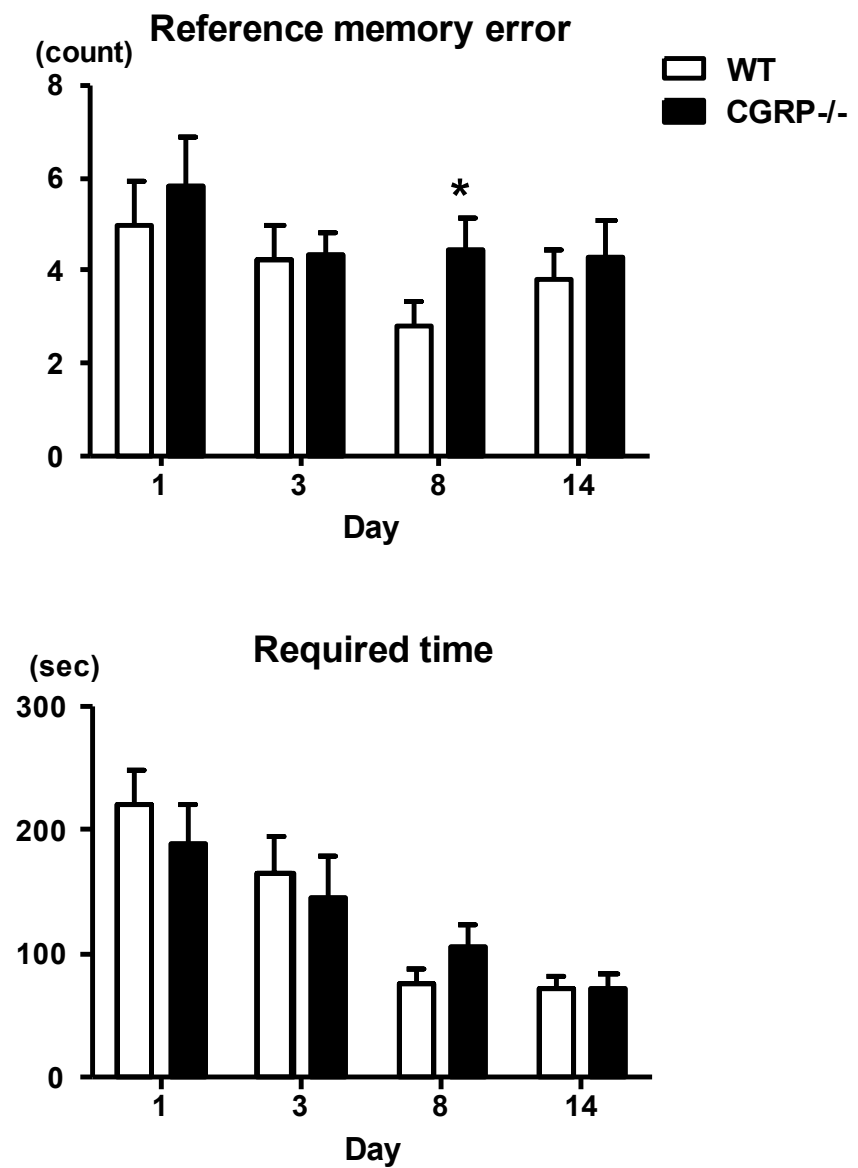

Figure 9 
A
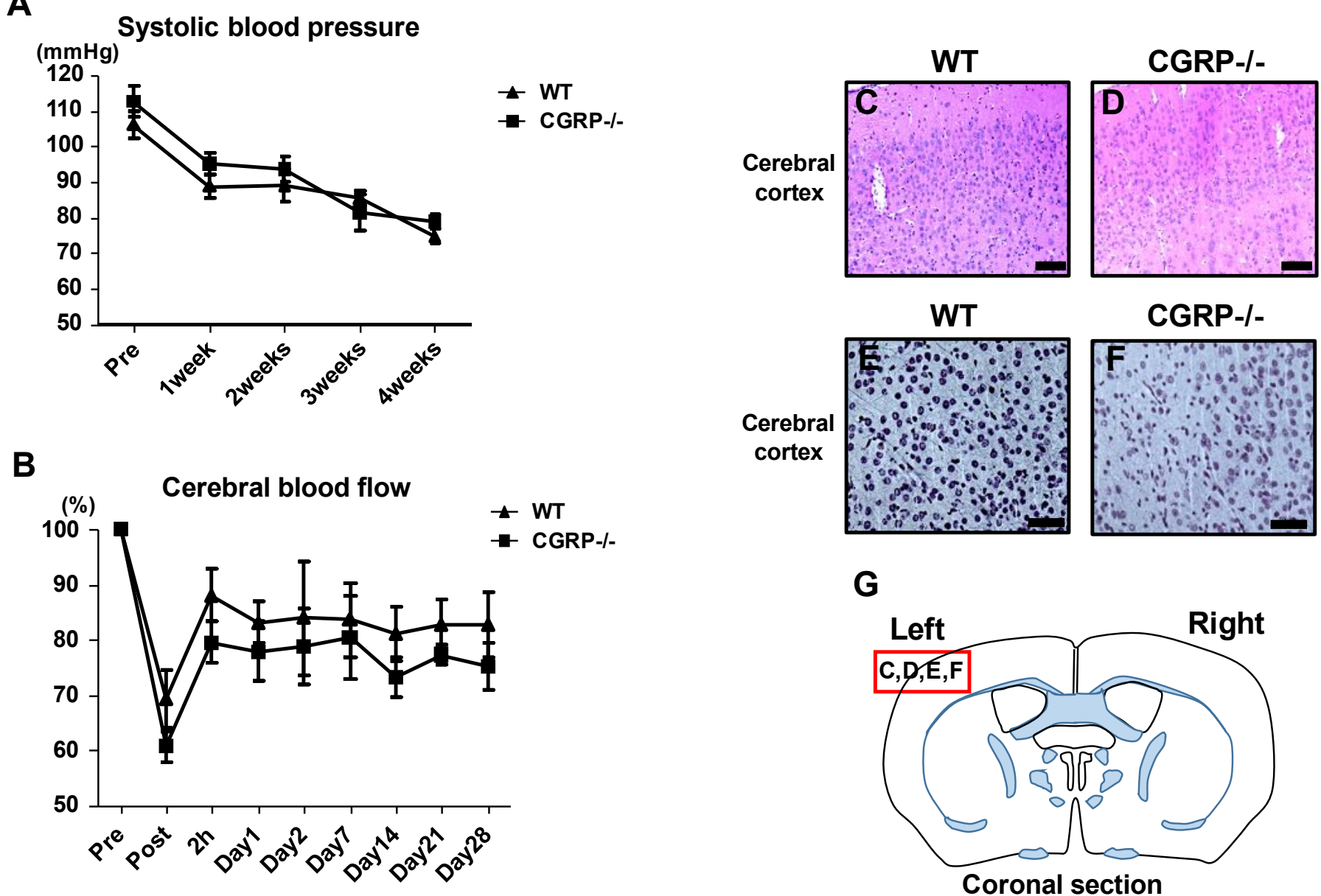

Figure 10

G

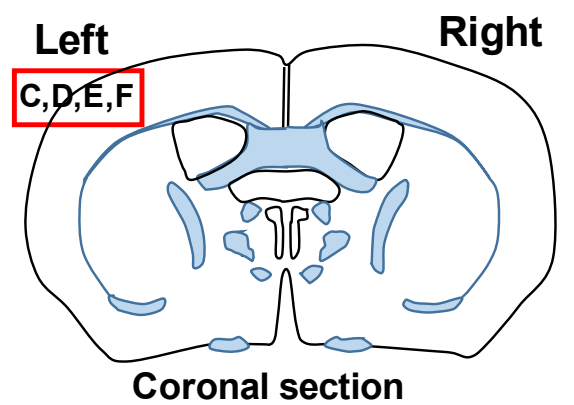

of the third ventricle level 
A $\quad(\mathrm{mmHg})$ Systolic blood pressure

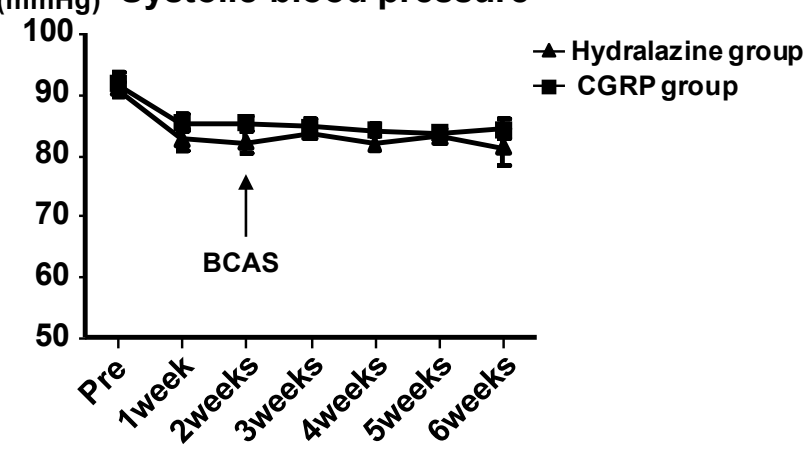

B

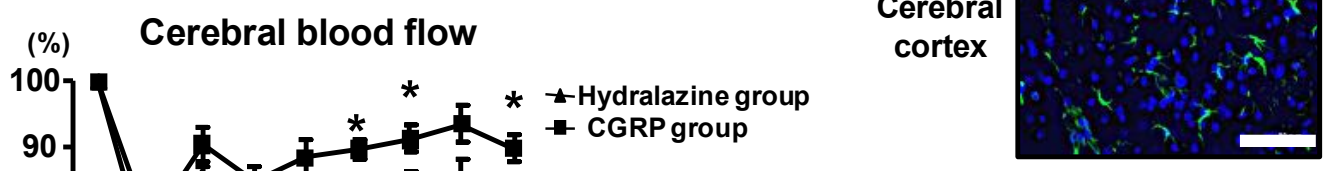

Callous corpus

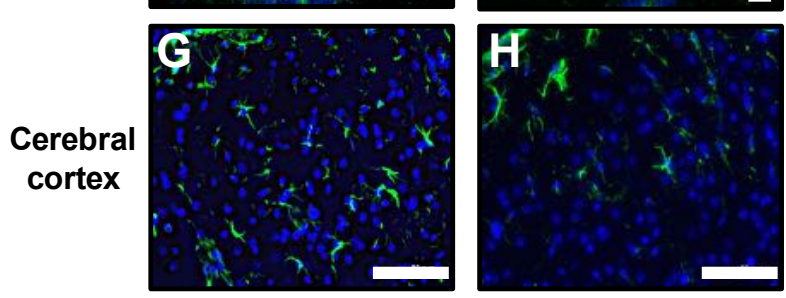

Hydralazine

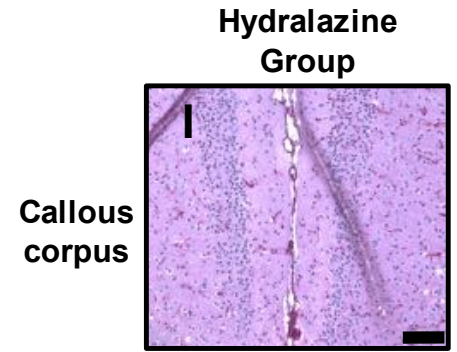

Hydralazine

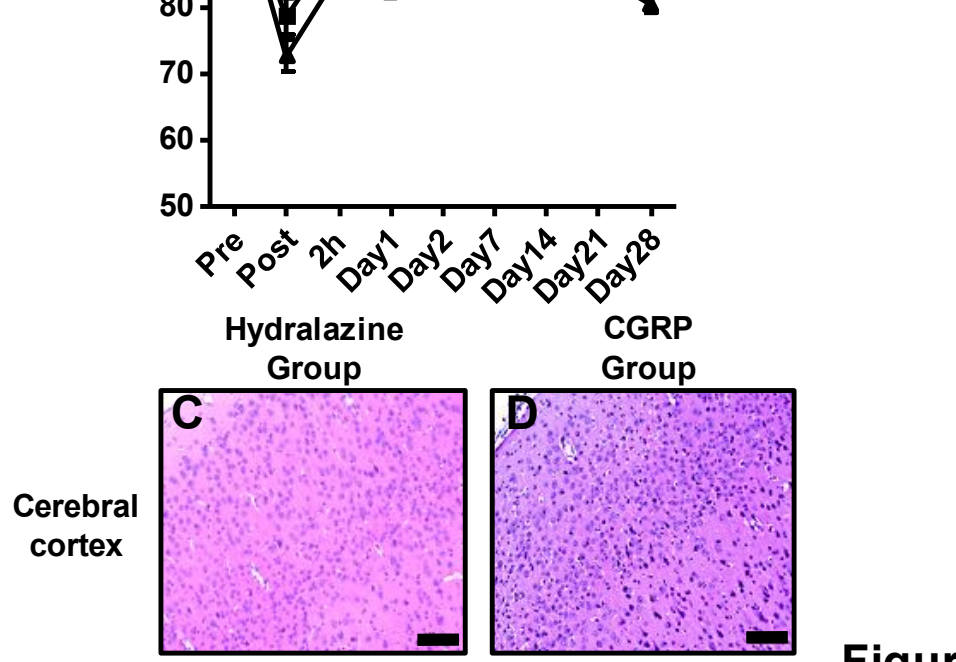

Hydralazine

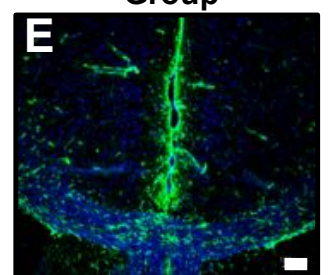

Figure 11

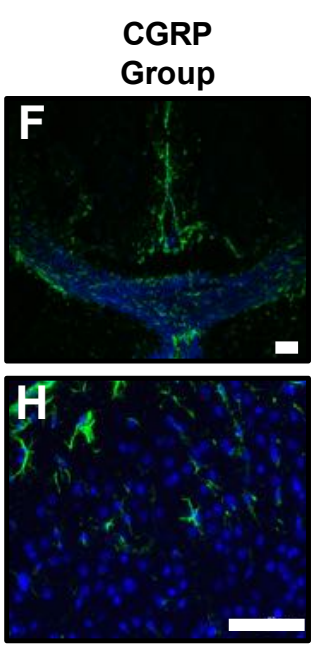

CGRP

Group

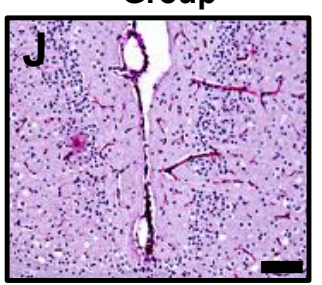

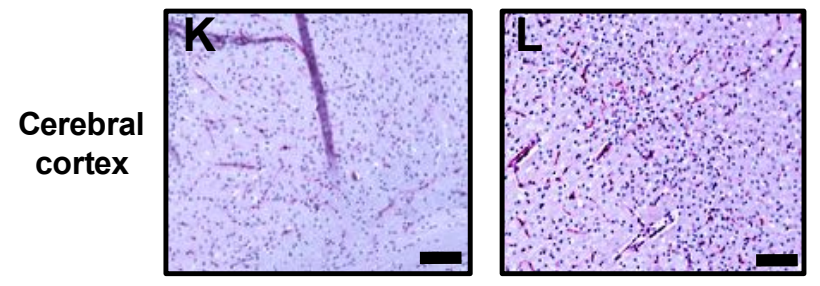


A

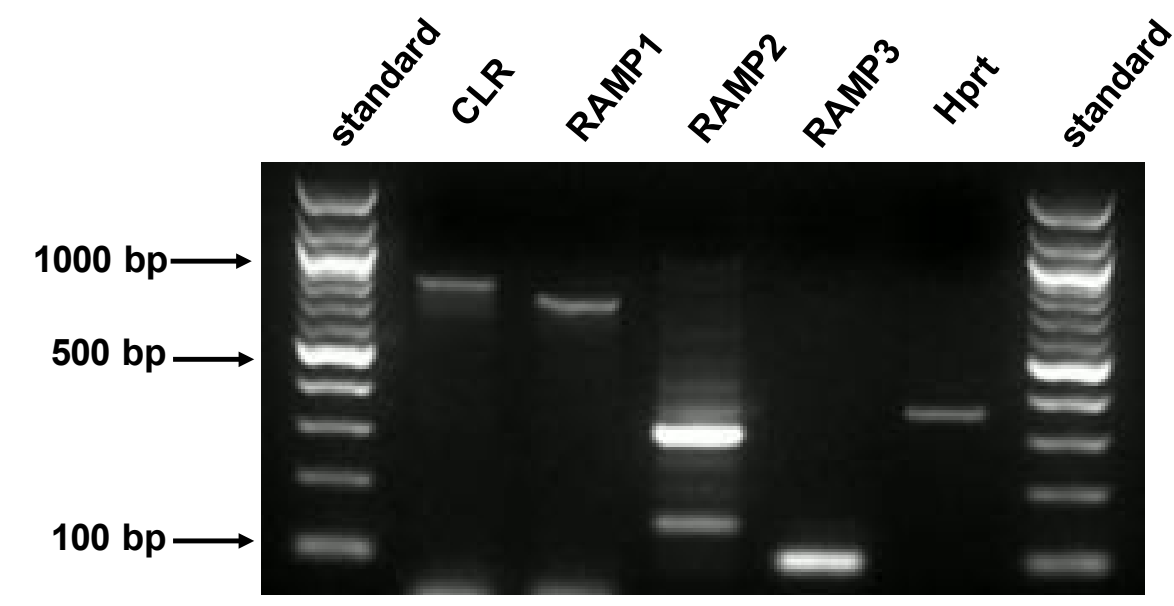

B

Cell viability

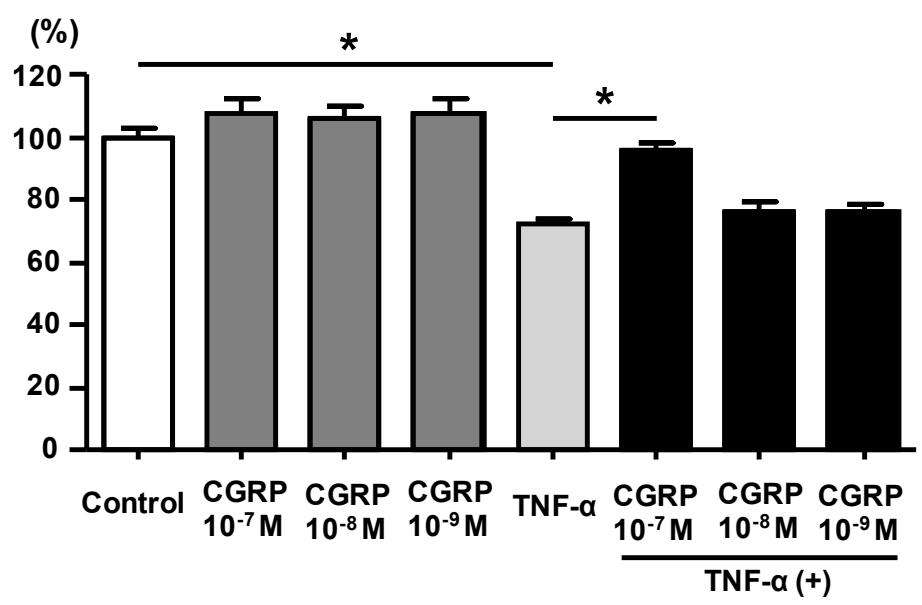

Figure 12 